\title{
SUPPLEMENT TO: ON FALSE DISCOVERY RATE THRESHOLDING FOR CLASSIFICATION UNDER SPARSITY
}

\author{
By Pierre Neuvial and Etienne Roquain \\ We provide proofs and complements for the paper [2]. We use the \\ notation and the equation numbering defined in [2].
}

\section{CONTENTS}

S-1 Generalization to some $\log$ concave densities . . . . . . . . . . . 1

S-2 Proofs for location and scale models . . . . . . . . . . . 4

S-2.1 Proof of $\left(\mathrm{A}\left(F_{m}, \tau_{m}\right)\right) \ldots \ldots \ldots \ldots$

S-2.2 Proof of Proposition $4.1 \ldots \ldots \ldots$

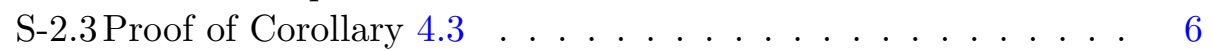

S-2.4Proof of Corollaries 4.4 and S-1.1 f . . . . . . . . . 7

S-2.5 Proof for Section $4.4 \ldots \ldots \ldots$

S-3 Calculations for some standard densities . . . . . . . . . . . . 11

S-3.1 Equivalents for $\alpha_{m}^{o p t}\left(\beta_{0}, C_{0}\right) \ldots \ldots \ldots \ldots \ldots 11$

S-3.2 Laplace scale model . . . . . . . . . . . . . . . . . 12

S-3.2.1 Additional oracle inequalities and a lower bound . . . 12

S-3.2.2 Proof of Proposition S-3.1 . . . . . . . . . . . . . . 13

S-3.2.3 Proof of Corollary S-3.2 . . . . . . . . . . . . . . 15

S-3.3 Laplace location model . . . . . . . . . . . . . . . . . 16

S-3.4Gaussian models . . . . . . . . . . . . . . . . . 16

S-4 Study of the weighted mis-classification risk . . . . . . . . . 17

S-5 Expressions for tails and quantiles . . . . . . . . . . . . . . 20

S-6 A sub-optimality result . . . . . . . . . . . . . . . . . . . . 21

S-7 Additional numerical experiments . . . . . . . . . . . . . . . . . . . . . . . . . 22

References......................... 22

Author's addresses . . . . . . . . . . . . . . . . . . 22

S-1. Generalization to some log concave densities. Many proofs in this supplementary file are stated in the more general case where the density $d$ is of the form

$$
d(x)=e^{-\phi(|x|)}
$$


for a known function $\phi$ satisfying

$(\mathrm{A}(\phi))$

$\phi: \mathbb{R}^{+} \rightarrow \mathbb{R}$ is $C^{1}$ increasing and convex on $\mathbb{R}^{+}$with $\int_{\mathbb{R}} e^{-\phi(|x|)} d x=1$.

Assumption $(\mathrm{A}(\phi))$ sets a condition on $d(x)=e^{-\phi(|x|)}$ slightly stronger than " $d$ is symmetric log concave". Namely, it also entails that $d(\cdot)$ is decreasing on $\mathbb{R}^{+}$. This is essential to get a monotonic likelihood ratio. Of course, we recover the case of $\zeta$-Subbotin density by letting $\phi(u)=$ $u^{\zeta} / \zeta+\log \left(L_{\zeta}\right)$. The corresponding upper tail distribution is now defined by $\bar{D}(u)=\int_{u}^{+\infty} e^{-\phi(|x|)} d x$.

Using this general density, the location and scale models presented in Section 1.2 of the main paper are still particular instances of the general $p$-value model defined in Section 2.1 of the main paper: in the scale model, we apply the standardization $p_{i}=2 \bar{D}\left(\left|X_{i}\right|\right)$, which yields $F_{m}(t)=2 \bar{D}\left(\bar{D}^{-1}(t / 2) / \sigma_{m}\right)$. We can check that if $\phi$ satisfies $(\mathrm{A}(\phi))$, then $F_{m}(t)=2 \bar{D}\left(\bar{D}^{-1}(t / 2) / \sigma_{m}\right)$ satisfies $\left(\mathrm{A}\left(F_{m}, \tau_{m}\right)\right)$, with $f_{m}\left(0^{+}\right)=+\infty$ and $f_{m}\left(1^{-}\right)<1$, see Section S-2.1. In the location model, we let $p_{i}=\bar{D}\left(X_{i}\right)$, which yields $F_{m}(t)=\bar{D}\left(\bar{D}^{-1}(t)-\right.$ $\left.\mu_{m}\right)$. For this case, $(\mathrm{A}(\phi))$ is not sufficient to ensure that $f_{m}=F_{m}^{\prime}$ is decreasing and we will use the following additional assumption on $\phi$ :

$\left(\mathrm{A}^{\prime}(\phi)\right)$

$\phi$ satisfies $(\mathrm{A}(\phi))$ and $\phi^{\prime}$ is increasing on $\mathbb{R}^{+}$with $\lim _{+\infty} \phi^{\prime}=+\infty$,

which ensures that $F_{m}(t)=\bar{D}\left(\bar{D}^{-1}(t)-\mu_{m}\right)$ satisfies $\left(\mathrm{A}\left(F_{m}, \tau_{m}\right)\right)$, with $f_{m}\left(0^{+}\right)=+\infty$ and $f_{m}\left(1^{-}\right)=0$, as proved in Section S-2.1.

Under this general model, we define the rates $r_{m}^{l o c}$ and $r_{m}^{s c}$ as follows:

$$
\begin{aligned}
r_{m}^{l o c} & =\phi^{\prime} \circ \phi^{-1}\left(\log \tau_{m}+\phi\left(\left|\bar{D}^{-1}\left(C_{m}\right)\right|\right)\right) ; \\
r_{m}^{s c} & =\left(\operatorname{Id} \times \phi^{\prime}\right) \circ \phi^{-1}\left(\log \tau_{m}+\phi\left(\bar{D}^{-1}\left(C_{m} / 2\right)\right)\right),
\end{aligned}
$$

where Id denotes the identity function, hence, $\left(\operatorname{Id} \times \phi^{\prime}\right)(x)=x \phi^{\prime}(x)$. Under (Sp), we easily check that the rates $r_{m}^{l o c}$ (resp., $r_{m}^{s c}$ ) tend to infinity, given that $\phi$ satisfies $\left(\mathrm{A}^{\prime}(\phi)\right)$ (resp., $(\mathrm{A}(\phi))$ ). Table $\mathrm{S}-1$ provides some useful calculations for $\phi$. They can be used to check that, in the case where it comes from a $\zeta$-Subbotin density, we have $r_{m}^{l o c}=\left(\zeta \log \tau_{m}+\left|\bar{D}^{-1}\left(C_{m}\right)\right|^{\zeta}\right)^{1-1 / \zeta}$ and $r_{m}^{s c}=\zeta \log \tau_{m}+\left(\bar{D}^{-1}\left(C_{m} / 2\right)\right)^{\zeta}$.

In the main paper, Proposition 4.1, (23) and (24) in Section 4.1, and Corollary 4.3 in Section 4.2 can be readily extended by considering a function $\phi$ satisfying $(\mathrm{A}(\phi))$ in the scale model or $\left(\mathrm{A}^{\prime}(\phi)\right)$ in the location model. 


\begin{tabular}{|c|c|}
\hline$\phi(u)$ & $u^{\zeta} / \zeta+\log L_{\zeta}$ \\
$\phi^{\prime}(u)$ & $u^{\zeta-1}$ \\
$\phi^{\prime} \circ \phi^{-1}(v)$ & $\left(\zeta v-\zeta \log L_{\zeta}\right)^{1-1 / \zeta}$ \\
$\phi^{-1}(v) \times \phi^{\prime} \circ \phi^{-1}(v)$ & $\zeta v-\zeta \log L_{\zeta}$ \\
$\phi^{\prime \prime}(u)$ & $(\zeta-1) u^{\zeta-2}$ \\
$\phi^{\prime \prime}(u) /\left(\phi^{\prime}(u)\right)^{2}$ & $(\zeta-1) u^{-\zeta}$ \\
\hline \multicolumn{2}{|c|}{ TABLE S-1 }
\end{tabular}

Some useful calculations for the $\zeta$-Subbotin density.

However, in order to extend the results of Section 4.3, we need to introduce the following additional assumptions on $\phi$ :

$(B(\phi))$ $\phi$ satisfies $(\mathrm{A}(\phi)), \phi$ is $C^{2}$ on $\mathbb{R}^{+}$with $\phi^{\prime \prime} /\left(\phi^{\prime}\right)^{2}$ non-increasing on $(0, \infty)$;

$(C(\psi))$ $\psi(x+o(x)) \sim \psi(x)$ and $\psi(x)=O(x)$ as $x \rightarrow+\infty$,

either for $\psi=\phi^{\prime} \circ \phi^{-1}$ (location) or $\psi=\left(\operatorname{Id} \times \phi^{\prime}\right) \circ \phi^{-1}$ (scale). Note that assuming (BP) and (Sp), we have $r_{m} \sim \psi\left(\log \tau_{m}\right)$ under $(C(\psi))$, either for $r_{m}=r_{m}^{l o c}$ and $\psi=\phi^{\prime} \circ \phi^{-1}$ or for $r_{m}=r_{m}^{s c}$ and $\psi=\left(\operatorname{Id} \times \phi^{\prime}\right) \circ \phi^{-1}$. Also, from Table S-1, when considering a $\zeta$-Subbotin density, Assumptions $(B(\phi))$ and $(C(\psi))$ both hold with $\psi=\phi^{\prime} \circ \phi^{-1}$ and $\psi=\left(\operatorname{Id} \times \phi^{\prime}\right) \circ \phi^{-1}$.

Then, Corollary 4.4 can be extended as follows:

Corollary S-1.1. Consider $d(x)=e^{-\phi(|x|)}$ for a function $\phi$ satisfying $(\mathrm{A}(\phi))$ in the scale model or $\left(\mathrm{A}^{\prime}(\phi)\right)$ in the location model. Let $\left(\tau_{m}, C_{m}\right) \in$ $(1, \infty) \times(0,1)$ be the parameters of the model. Let $r_{m}$ and $\psi(\cdot)$ be defined as follows:

- in the location model, $r_{m}=r_{m}^{l o c}$ defined by (21) and $\psi=\phi^{\prime} \circ \phi^{-1}$;

- in the scale model, $r_{m}=r_{m}^{s c}$ defined by $(22)$ and $\psi=\left(I d \times \phi^{\prime}\right) \circ \phi^{-1}$.

Assume that (BP) and (Sp) hold. Consider the BFDR threshold $t_{m}^{\star}$ at a level $\alpha_{m} \in(0,1)$. Then the following holds:

(i) The BFDR threshold $t_{m}^{\star}$ is asymptotically optimal if

$$
\alpha_{m} \rightarrow 0 \text { and } \log \alpha_{m}=o\left(r_{m}\right)
$$

in which case it is asymptotically optimal at rate $\rho_{m}=\alpha_{m}+\left(\log \left(\alpha_{m}^{-1} / r_{m}\right)\right)_{+} / r_{m}$. Additionally, if $\phi$ satisfies $(B(\phi))$ and $\psi$ satisfies $(C(\psi))$, the BFDR threshold $t_{m}^{\star}$ is asymptotically optimal if and only if (S-3) holds.

imsart-aos ver. 2012/08/16 file: AOS1042_supp.tex date: August 22, 2012 
(ii) Further assume that there exists $\lambda>0$ such that $\psi(x)=O\left(e^{\lambda x}\right)$ for $x \rightarrow+\infty$ and that the sparsity regime $\tau_{m}$ satisfies

$$
m / \tau_{m} \geq\left(\log \tau_{m}\right)^{1+\theta} \text { for some } \theta>0 ; \quad \text { or } m / \tau_{m} \rightarrow l \in(0,+\infty) .
$$

Then, the FDR threshold $\hat{t}_{m}^{F D R}$ at a level $\alpha_{m}$ satisfying (S-3) is asymptotically optimal at rate $\rho_{m}=\alpha_{m}+\left(\log \left(\alpha_{m}^{-1} / r_{m}\right)\right)_{+} / r_{m}$.

\section{S-2. Proofs for location and scale models.}

S-2.1. Proof of $\left(\mathrm{A}\left(F_{m}, \tau_{m}\right)\right)$. First, assume $\left(\mathrm{A}^{\prime}(\phi)\right)$ and consider the location model: we easily check that

$$
f_{m}(t)=\exp \left\{\phi\left(\left|\bar{D}^{-1}(t)\right|\right)-\phi\left(\left|\bar{D}^{-1}(t)-\mu_{m}\right|\right)\right\} .
$$

Thus for $t$ such that $\bar{D}^{-1}(t)>\mu_{m}$, we have $\log f_{m}(t)=\phi\left(\bar{D}^{-1}(t)\right)-$ $\phi\left(\bar{D}^{-1}(t)-\mu_{m}\right) \geq \phi^{\prime}\left(\bar{D}^{-1}(t)-\mu_{m}\right) \mu_{m}$, by using the convexity of $\phi$. Since $\lim _{+\infty} \phi^{\prime}=+\infty$, we obtain $f_{m}\left(0^{+}\right)=+\infty$. For $t$ such that $\bar{D}^{-1}(t)<0$, $-\log f_{m}(t)=\phi\left(-\bar{D}^{-1}(t)+\mu_{m}\right)-\phi\left(-\bar{D}^{-1}(t)\right) \geq \phi^{\prime}\left(-\bar{D}^{-1}(t)\right) \mu_{m}$. Hence we also have $f_{m}\left(1^{-}\right)=0$. Furthermore, $f_{m}$ is decreasing because $\phi$ is strictly convex and increasing under $\left(\mathrm{A}^{\prime}(\phi)\right)$.

Second, assume $(\mathrm{A}(\phi))$ and consider the scale model. In this case, we have

$$
f_{m}(t)=\sigma_{m}^{-1} \exp \left\{\phi\left(\bar{D}^{-1}(t / 2)\right)-\phi\left(\bar{D}^{-1}(t / 2) / \sigma_{m}\right)\right\} .
$$

Thus $f_{m}(1)=\sigma_{m}^{-1}<1$. By using the convexity of $\phi$, we have $\log \left(\sigma_{m} f_{m}(t)\right)=$ $\phi\left(\bar{D}^{-1}(t / 2)\right)-\phi\left(\bar{D}^{-1}(t / 2) / \sigma_{m}\right) \geq\left(1-\sigma_{m}^{-1}\right) \bar{D}^{-1}(t / 2) \phi^{\prime}\left(\bar{D}^{-1}(t / 2) / \sigma_{m}\right)$. Hence $f_{m}\left(0^{+}\right)=+\infty$. Finally, $f_{m}$ is decreasing because $\phi$ is convex.

\section{S-2.2. Proof of Proposition 4.1.}

Lemma S-2.1. Consider the location model with a density $d(x)=e^{-\phi(|x|)}$ for a function $\phi$ satisfying $\left(\mathrm{A}^{\prime}(\phi)\right)$. Then we have for any $m \geq 2$,

$$
\begin{aligned}
\mu_{m} & =\phi^{-1}\left(\log \tau_{m}+\phi\left(\left|\bar{D}^{-1}\left(C_{m}\right)\right|\right)\right)-\bar{D}^{-1}\left(C_{m}\right) \\
t_{m}^{B} & \leq \frac{d\left(\bar{D}^{-1}\left(C_{m}\right)\right)}{\tau_{m} r_{m}^{l o c}} \\
t_{m}^{B} & \geq \frac{d\left(\bar{D}^{-1}\left(C_{m}\right)\right)}{\tau_{m} r_{m}^{l o c}}\left(1+\frac{\phi^{\prime \prime}}{\phi^{\prime 2}}\left(\bar{D}^{-1}\left(C_{m}\right)+\mu_{m}\right)\right)^{-1} \text { if }(B(\phi)) \text { holds }
\end{aligned}
$$

$$
R_{m}\left(t_{m}^{B}\right) \leq \pi_{1, m}\left(\frac{d\left(\bar{D}^{-1}\left(C_{m}\right)\right)}{r_{m}^{l o c}}+1-C_{m}\right) .
$$


If (BP) and (Sp) hold, we have $t_{m}^{B}=O\left(\pi_{1, m} / r_{m}^{l o c}\right)$ and $R_{m}\left(t_{m}^{B}\right) \sim \pi_{1, m}(1-$ $\left.C_{m}\right)$. If additionally $(B(\phi))$ holds, we have $\tau_{m} t_{m}^{B} \sim d\left(\bar{D}^{-1}\left(C_{m}\right)\right) / r_{m}^{l o c}$.

Proof. First, since $C_{m}=F_{m}\left(t_{m}^{B}\right)=\bar{D}\left(\bar{D}^{-1}\left(t_{m}^{B}\right)-\mu_{m}\right)$, we have

$$
\tau_{m}=f_{m}\left(t_{m}^{B}\right)=\exp \left\{\phi\left(\left|\bar{D}^{-1}\left(C_{m}\right)+\mu_{m}\right|\right)-\phi\left(\left|\bar{D}^{-1}\left(C_{m}\right)\right|\right)\right\}
$$

Since $\tau_{m} \geq 1$ and $\phi$ is increasing, we get $\left|\bar{D}^{-1}\left(C_{m}\right)+\mu_{m}\right| \geq\left|\bar{D}^{-1}\left(C_{m}\right)\right|$. Then, we note that for any $a>0$ and $b \in \mathbb{R},|b+a| \geq|b|$ holds only if $a+b \geq 0$. This provides that $\bar{D}^{-1}\left(C_{m}\right)+\mu_{m} \geq 0$ and yields (S-5). Next, we have $t_{m}^{B}=F_{m}^{-1}\left(C_{m}\right)=\bar{D}\left(\bar{D}^{-1}\left(C_{m}\right)+\mu_{m}\right)$. First, using (S-44), we obtain that $t_{m}^{B} \leq d\left(\bar{D}^{-1}\left(C_{m}\right)+\mu_{m}\right) / \phi^{\prime}\left(\bar{D}^{-1}\left(C_{m}\right)+\mu_{m}\right)$. Since $\tau_{m} d\left(\bar{D}^{-1}\left(C_{m}\right)+\mu_{m}\right)=$ $d\left(\bar{D}^{-1}\left(C_{m}\right)\right)$, we obtain (S-6) and then (S-8). Second, if $\phi$ satisfies $(B(\phi))$ we can apply (S-46) to get (S-7). To finish the proof, we only have to prove that $(B(\phi))$ implies that $\lim _{\infty} \phi^{\prime \prime} / \phi^{\prime 2}=0$; if $(B(\phi))$ holds then $\lim _{\infty} \phi^{\prime}$ exists in $(0, \infty]$ and thus $h=-1 / \phi^{\prime}$ is non-decreasing concave with a finite limit in $\infty$. This entails that $h^{\prime}=\phi^{\prime \prime} /\left(\phi^{\prime}\right)^{2}$ tends to zero in $\infty$.

Lemma S-2.2. Consider the scale model with a density $d(x)=e^{-\phi(|x|)}$ for a function $\phi$ satisfying $(\mathrm{A}(\phi))$. Then, we have for any $m \geq 2$,

$$
\log \tau_{m}=-\log \sigma_{m}+\phi\left(\bar{D}^{-1}\left(C_{m} / 2\right) \sigma_{m}\right)-\phi\left(\bar{D}^{-1}\left(C_{m} / 2\right)\right)
$$

$$
\sigma_{m} \geq \phi^{-1}\left(\log \tau_{m}+\phi\left(\bar{D}^{-1}\left(C_{m} / 2\right)\right)\right) / \bar{D}^{-1}\left(C_{m} / 2\right)
$$

$$
t_{m}^{B} \leq \frac{\tau_{m}^{-1} 2 d\left(\bar{D}^{-1}\left(C_{m} / 2\right)\right)}{\sigma_{m} \phi^{\prime}\left(\bar{D}^{-1}\left(C_{m} / 2\right) \sigma_{m}\right)}
$$

$$
t_{m}^{B} \geq \frac{\tau_{m}^{-1} 2 d\left(\bar{D}^{-1}\left(C_{m} / 2\right)\right)}{\sigma_{m} \phi^{\prime}\left(\bar{D}^{-1}\left(C_{m} / 2\right) \sigma_{m}\right)}\left(1+\frac{\phi^{\prime \prime}}{\phi^{\prime 2}}\left(\bar{D}^{-1}\left(C_{m} / 2\right) \sigma_{m}\right)\right)^{-1} \text { if }(B(\phi)) \text { holds }
$$

$R_{m}\left(t_{m}^{B}\right) \leq \pi_{1, m}\left(\frac{2 \bar{D}^{-1}\left(C_{m} / 2\right) d\left(\bar{D}^{-1}\left(C_{m} / 2\right)\right)}{r_{m}^{s c}}+1-C_{m}\right)$.

In particular, if (BP) and (Sp) hold, we have $\log \tau_{m} \sim \phi\left(\bar{D}^{-1}\left(C_{m} / 2\right) \sigma_{m}\right)$, $t_{m}^{B}=O\left(\pi_{1, m} / r_{m}^{s c}\right)$ and $R_{m}\left(t_{m}^{B}\right) \sim \pi_{1, m}\left(1-C_{m}\right)$. If additionally $(B(\phi))$ holds, we have $\tau_{m} t_{m}^{B} \sim \frac{2 d\left(\bar{D}^{-1}\left(C_{m} / 2\right)\right)}{\sigma_{m} \phi^{\prime}\left(\bar{D}^{-1}\left(C_{m} / 2\right) \sigma_{m}\right)}$. 
Proof. First, since $C_{m}=F_{m}\left(t_{m}^{B}\right)=2 \bar{D}\left(\bar{D}^{-1}\left(t_{m}^{B} / 2\right) / \sigma_{m}\right)$, we have

$$
\tau_{m}=f_{m}\left(t_{m}^{B}\right)=\sigma_{m}^{-1} \exp \left\{\phi\left(\bar{D}^{-1}\left(C_{m} / 2\right) \sigma_{m}\right)-\phi\left(\bar{D}^{-1}\left(C_{m} / 2\right)\right)\right\}
$$

and thus (S-9) holds. Since $\log \sigma_{m}>0$, we get (S-10). Next, using (S-44), we obtain that

$$
t_{m}^{B}=F_{m}^{-1}\left(C_{m}\right)=2 \bar{D}\left(\bar{D}^{-1}\left(C_{m} / 2\right) \sigma_{m}\right) \leq \frac{2 d\left(\bar{D}^{-1}\left(C_{m} / 2\right) \sigma_{m}\right)}{\phi^{\prime}\left(\bar{D}^{-1}\left(C_{m} / 2\right) \sigma_{m}\right)} .
$$

Since we have $\sigma_{m} \tau_{m} d\left(\bar{D}^{-1}\left(C_{m} / 2\right) \sigma_{m}\right)=d\left(\bar{D}^{-1}\left(C_{m} / 2\right)\right)$ by (S-9) and by (S-10), we obtain (S-11), and then (S-13). Expression (S-12) is derived similarly by using (S-46). Finally, if (BP) and (Sp) hold, we obtain $\log \tau_{m} \sim$ $\phi\left(\bar{D}^{-1}\left(C_{m} / 2\right) \sigma_{m}\right)$ by applying (S-9) and by noting that $\phi(x)-\log x \sim \phi(x)$ as $x$ tends to infinity because $\phi(x) / x \geq \phi^{\prime}(1)>0$ for $x \geq 1$. The remaining statements are then straightforward.

S-2.3. Proof of Corollary 4.3. Proof for (i): from Theorem 3.1 (i), to show (26), we only have to prove that $\gamma_{m}=\left(C_{m}-F_{m}\left(\Psi_{m}^{-1}\left(q_{m} \tau_{m}\right)\right)\right)_{+}$satisfies

$$
\gamma_{m} \leq K_{m}\left(\log \left(q_{m} / q_{m}^{o p t}\right)-\log \nu\right)_{+} / r_{m}
$$

When $q_{m} \leq q_{m}^{\text {opt }}$, this is trivial because $\gamma_{m}=0$. Assume now $q_{m}>q_{m}^{\text {opt }}$ so that $\gamma_{m}=C_{m}-F_{m}\left(\Psi_{m}^{-1}\left(q_{m} \tau_{m}\right)\right)=F_{m}\left(\Psi_{m}^{-1}\left(q_{m}^{o p t} \tau_{m}\right)\right)-F_{m}\left(\Psi_{m}^{-1}\left(q_{m} \tau_{m}\right)\right) \geq 0$. To prove (S-14), we apply Lemma S-2.3 (below) with $\eta_{m}=C_{m}(1-\nu)$ to get that,

$$
\begin{aligned}
\log \left(\frac{\left.\Psi_{m} \circ F_{m}^{-1}\left(\nu C_{m}\right)\right)}{\Psi_{m} \circ F_{m}^{-1}\left(C_{m}\right)}\right) & \geq \log \nu+\frac{C_{m}(1-\nu)}{K_{m}} r_{m} \\
& \geq \log \left(q_{m} / q_{m}^{o p t}\right),
\end{aligned}
$$

where the last inequality holds by assumption. We thus obtain $\gamma_{m} \leq C_{m}$ (1$\nu$ ) by inverting (S-15) because $q_{m} / q_{m}^{o p t}=\frac{\Psi_{m} \circ F_{m}^{-1}\left(C_{m}-\gamma_{m}\right)}{\Psi_{m} \circ F_{m}^{-1}\left(C_{m}\right)}$. We can thus apply Lemma S-2.3 once again, this time for $\eta_{m}=\gamma_{m}$, we obtain

$$
\log \left(\frac{q_{m}}{q_{m}^{o p t}}\right) \geq \log \nu+\frac{\gamma_{m}}{K_{m}} r_{m}
$$

This implies (S-14).

Proof for (ii): We apply Theorem 3.2. Let us prove (27) for $a=1$. Let $q_{m}^{\varepsilon}=\left(\alpha_{m} \pi_{0, m}(1-\varepsilon)\right)^{-1}-1 \leq\left(\alpha_{m} \pi_{0, m}(1-\varepsilon)\right)^{-1}$ and $\gamma_{m}^{\varepsilon}=\left(C_{m}-\right.$ $\left.F_{m}\left(\Psi_{m}^{-1}\left(q_{m}^{\varepsilon} \tau_{m}\right)\right)\right)_{+}$. From the same reasoning as for (i) above, we obtain 
$\gamma_{m}^{\varepsilon} \leq C_{m}(1-\nu)$ and $\gamma_{m}^{\varepsilon} \leq K_{m}\left(\log \left(q_{m}^{\varepsilon} / q_{m}^{o p t}\right)-\log \nu\right)_{+} / r_{m}$ as soon as $r_{m} \geq \frac{K_{m}}{C_{m}(1-\nu)}\left(\log \left(q_{m}^{\varepsilon} / q_{m}^{o p t}\right)-\log \nu\right)$. This yields $(27)$ in the case $a=1$.

Now let us prove $(27)$ for $a=2$. First note that $\alpha_{m} / m=\Psi_{m}^{-1}\left(q_{m}^{\prime} \tau_{m}\right)$ where we let $q_{m}^{\prime}=\tau_{m}^{-1} m \alpha_{m}^{-1} F_{m}\left(\alpha_{m} / m\right)$. Hence, $\gamma_{m}^{\prime}=\left(C_{m}-F_{m}\left(\alpha_{m} / m\right)\right)_{+}=$ $\left(C_{m}-F_{m}\left(\Psi_{m}^{-1}\left(q_{m}^{\prime} \tau_{m}\right)\right)\right)_{+}$. Assume $\alpha_{m} / m \leq t_{m}^{B}$ (otherwise $\gamma_{m}^{\prime}=0$ and the result is trivial). From the same reasoning as for (i), we can show $\gamma_{m}^{\prime} \leq K_{m}\left(\log \left(q_{m}^{\prime} / q_{m}^{o p t}\right)-\log \nu\right)_{+} / r_{m}$. Hence the result comes from $q_{m}^{\prime} \leq$ $\tau_{m}^{-1} m \alpha_{m}^{-1} C_{m}$ because $F_{m}\left(\alpha_{m} / m\right) \leq F_{m}\left(t_{m}^{B}\right)=C_{m}$.

We now state and prove Lemma S-2.3.

Lemma S-2.3. Consider the setting of Corollary 4.3. Let $\eta_{m}$ be such that $0 \leq \eta_{m} \leq C_{m}(1-\nu)$, for some $\nu \in(0,1)$. Then, we have

$$
\log \left(\frac{\Psi_{m} \circ F_{m}^{-1}\left(C_{m}-\eta_{m}\right)}{\Psi_{m} \circ F_{m}^{-1}\left(C_{m}\right)}\right) \geq \log \nu+\frac{\eta_{m} r_{m}}{K_{m}} .
$$

Proof. Let us prove the location model (the scale case is similar). Let us fist note that the function $-\log \bar{D}$ is increasing on $\mathbb{R}$ and also convex on $(0,+\infty)$, because its second derivative on $(0,+\infty)$ is $d \times\left(-\bar{D} \phi^{\prime}+d\right) /(\bar{D})^{2}$ which is non-negative by (S-44). Next, since $\Psi_{m} \circ F_{m}^{-1}(t)=t / \bar{D}\left(\bar{D}^{-1}(t)+\right.$ $\left.\mu_{m}\right)$, we have

$$
\begin{aligned}
& \log \left(\frac{\Psi_{m} \circ F_{m}^{-1}\left(C_{m}-\eta_{m}\right)}{\Psi_{m} \circ F_{m}^{-1}\left(C_{m}\right)}\right) \\
& =\log \left(\frac{C_{m}-\eta_{m}}{C_{m}}\right)-\log \left(\frac{\bar{D}\left(\bar{D}^{-1}\left(C_{m}-\eta_{m}\right)+\mu_{m}\right)}{\bar{D}\left(\bar{D}^{-1}\left(C_{m}\right)+\mu_{m}\right)}\right) \\
& \geq \log \nu+\left(\bar{D}^{-1}\left(C_{m}-\eta_{m}\right)-\bar{D}^{-1}\left(C_{m}\right)\right) \phi^{\prime}\left(\bar{D}^{-1}\left(C_{m}\right)+\mu_{m}\right)
\end{aligned}
$$

by using that $\bar{D}^{-1}\left(C_{m}\right)+\mu_{m}>0$ (as stated in Lemma S-2.1), the convexity of $-\log \bar{D}$ on $(0,+\infty)$ and that the derivative $d / \bar{D}$ of $-\log \bar{D}$ on $(0,+\infty)$ satisfies $d / \bar{D} \geq \phi^{\prime}$ (by using again (S-44)). Finally, since $-\bar{D}^{-1}$ is increasing and of derivative $1 / d\left(\bar{D}^{-1}(\cdot)\right) \geq 1 / d(0)$, we have $\bar{D}^{-1}\left(C_{m}-\eta_{m}\right)-\bar{D}^{-1}\left(C_{m}\right) \geq$ $\eta_{m} / d(0)$. Finally note that from $(\mathrm{S}-5)$, we have $\phi^{\prime}\left(\bar{D}^{-1}\left(C_{m}\right)+\mu_{m}\right)=r_{m}^{l o c}$, which gives the result.

S-2.4. Proof of Corollaries 4.4 and S-1.1. Corollary 4.4 is a special case of Corollary S-1.1 in the Subbotin case. Let us prove Corollary S-1.1. Let us start by proving (i). First note that $r_{m} \rightarrow \infty$ as soon as $m \rightarrow \infty$. The first claim in (i) easily derives from (26), because $r_{m}$ is larger than 
$\frac{K_{m}}{C_{m}(1-\nu)}\left(\log q_{m}-\log \nu\right)$ for large $m$ if (S-3) holds and because $q_{m}^{o p t} \geq 1$. This entails that $t_{m}^{\star}$ is asymptotically optimal at rate $\rho_{m}=\alpha_{m}+\left[\left(\log \left(\alpha_{m}^{-1} / q_{m}^{o p t}\right)\right)_{+}+\right.$ $1] / r_{m}$. Since $1 / q_{m}^{o p t}=O\left(1 / r_{m}\right)$ by $(24)$ and since we have for all $x, y>0$, $1 / x+(\log (x / y))_{+} / y \geq 1 / y$, we end up with the desired rate. Next, we prove the second claim only in the case of the location model (the scale case in similar). Assume $(B(\phi))$ and $(C(\psi))$ for $\psi=\phi^{\prime} \circ \phi^{-1}$. From above, we only have to prove that $t_{m}^{\star}$ is not asymptotically optimal whenever (S-3) is not fulfilled. For this, we apply Theorem 3.1 (ii) and we prove that any regime for which (S-3) is violated leads to (18). By considering a subsequence, we can assume that $C_{m}$ tends to some constant $C \in(0,1)$. It is thus sufficient to prove that $C^{\star}<C$ for $C^{\star}=\lim \sup _{m}\left\{\left(1-q_{m}^{-1}\right)_{+} F_{m}\left(q_{m}^{-1} \tau_{m}^{-1}\right)\right\}$.

Let us first note that the following holds from $(\mathrm{S}-5)$ :

$$
\begin{aligned}
F_{m}\left(q_{m}^{-1} \tau_{m}^{-1}\right) & =\bar{D}\left(\bar{D}^{-1}\left(q_{m}^{-1} \tau_{m}^{-1}\right)-\mu_{m}\right) \\
& =\bar{D}\left(\bar{D}^{-1}\left(C_{m}\right)+\kappa_{m}\right)
\end{aligned}
$$

where $q_{m}=\alpha_{m}^{-1}-1$ and where we let $\kappa_{m}=\bar{D}^{-1}\left(q_{m}^{-1} \tau_{m}^{-1}\right)-\phi^{-1}\left(\log \tau_{m}+\right.$ $\left.\phi\left(\left|\bar{D}^{-1}\left(C_{m}\right)\right|\right)\right)$. Next, from $(B(\phi))$ and (S-48), there exists a constant $K>0$ such that for any $t$ small enough, $\bar{D}^{-1}(t) \geq \phi^{-1}\left(\log 1 / t-\log \circ \phi^{\prime} \circ \phi^{-1}(\log 1 / t)-\right.$ $\log K)$. Also, from Appendix S-6, we can always assume that $q_{m}$ is bounded away from 0 and thus $q_{m}^{-1} \tau_{m}^{-1}$ necessarily converges to zero. Moreover, $\phi^{-1}$ is increasing and concave on $\mathbb{R}^{+}$, of derivative $1 / \phi^{\prime} \circ \phi^{-1}$. Thus we can write for $m$ large enough,

$$
\begin{aligned}
\kappa_{m} & \geq \phi^{-1}\left(\log \tau_{m}+\iota_{m}\right)-\phi^{-1}\left(\log \tau_{m}+\phi\left(\left|\bar{D}^{-1}\left(C_{m}\right)\right|\right)\right) \\
& \geq \frac{\iota_{m}-\phi\left(\left|\bar{D}^{-1}\left(C_{m}\right)\right|\right.}{\phi^{\prime} \circ \phi^{-1}\left(\left(\log \tau_{m}+\iota_{m}\right) \vee\left(\log \tau_{m}+\phi\left(\left|\bar{D}^{-1}\left(C_{m}\right)\right|\right)\right)\right)},
\end{aligned}
$$

where $\iota_{m}=\log q_{m}-\log \circ \phi^{\prime} \circ \phi^{-1}\left(\log \tau_{m} q_{m}\right)-\log K$. We now use the latter bound in order to prove $C^{\star}<C$ in any regime for which (S-3) is violated.

- if $\alpha_{m}$ does not converges to 0: by considering a subsequence, there is $\alpha_{-} \in(0,1)$ such that $\alpha_{m}>\alpha_{-}$for $m$ large enough. Hence $\log q_{m}$ is bounded and we can use $(C(\psi))$ to show that $\kappa_{m} \sim \frac{-\log \circ \phi^{\prime} \circ \phi^{-1}\left(\log \tau_{m} q_{m}\right)}{\phi^{\prime} \circ \phi^{-1}\left(\log \left(\tau_{m} q_{m}\right)\right)}$ tends to zero. This implies that $C^{\star} \leq \lim \sup _{m}\left\{\left(1-q_{m}^{-1}\right)_{+}\right\} C \leq$ $\left(1-\alpha_{-}\right) C<C$.

- if $\alpha_{m} \rightarrow 0$ and $\left(\log q_{m}\right) / r_{m}^{l o c}$ does not converges to zero: by considering a subsequence, $\left(\log q_{m}\right) / r_{m}^{l o c}$ converges to some $l \in(0,+\infty]$.

imsart-aos ver. 2012/08/16 file: AOS1042_supp.tex date: August 22, 2012 
First, if $\log q_{m}=o\left(\log \tau_{m}\right)$, we can use $(C(\psi))$ to show $\liminf { }_{m} \kappa_{m} \geq$ $\liminf \operatorname{ing}_{m} \frac{\log q_{m}}{r_{m}^{l o c}}=l$. Second, if $\left(\log q_{m}\right) /\left(\log \tau_{m}\right)$ does not converges to zero, it is larger than $\delta \in(0,+\infty)$ for $m$ large enough (by considering a subsequence). Hence, we have $\log \tau_{m} \leq \delta^{-1} \log q_{m}$ for $m$ large enough, which entails $\liminf \operatorname{in}_{m} \kappa_{m} \geq \liminf _{m} \frac{\log q_{m}}{\phi^{\prime} \circ \phi^{-1}\left(\left(\delta^{-1}+1\right) \log q_{m}\right)}$. Moreover, the latter is bounded away from zero because $\phi^{\prime} \circ \phi^{-1}\left(\left(\delta^{-1}+1\right) \log q_{m}\right)=$ $O\left(\log q_{m}\right)$ by using $(C(\psi))$. Finally, in any case, we obtain that $\lim \inf _{m} \kappa_{m}>$ 0 and thus $C^{\star}=\bar{D}\left(\bar{D}^{-1}(C)+\liminf _{m} \kappa_{m}\right)<C$.

This concludes the proof for (i).

Let us now prove (ii). First, we consider the sparsity regime where $m / \tau_{m} \geq$ $(\log m)^{1+\theta}$ for some $\theta>0$. This condition implies that for any $\kappa>0$, $e^{-\kappa m / \tau_{m}}$ tends to zero faster than any power function $\tau_{m}^{-\lambda}, \lambda>0$. In particular, since by assumption $\Psi(x)=O\left(e^{\lambda x}\right)$ for $x \rightarrow+\infty, e^{-\kappa m / \tau_{m}}$ converges to zero faster than $1 / r_{m}$. In the second sparsity regime where $m / \tau_{m} \rightarrow l \in(0,+\infty)$, we have $m / \tau_{m}$ which is a bounded sequence. Finally, in any of the two sparsity regimes, the result follows from Corollary 4.3 (ii), because $R_{m}\left(t_{m}^{B}\right) \sim \tau_{m}^{-1}(1-C)$ and $\pi_{1, m} \gg \alpha_{m} / m$ (the rate is deduced by the same reasoning as in the proof of item (i) above).

\section{S-2.5. Proof for Section 4.4.}

Corollary S-2.4. Consider a $\zeta$-Subbotin location model with $\zeta>1$ and let $\left(\tau_{m}, C_{m}\right) \in(1, \infty) \times(0,1)$ be the parameters of the model. Assume $\tau_{m}=$ $m^{\beta}, \beta \in(0,1]$, choose $q_{m}=q_{m}^{o p t}\left(\beta_{0}, C_{0}\right)$ and consider $\rho_{m}=(\log m)^{1-1 / \zeta}$. Assume (BP) with some $0<C_{-} \leq C_{+}<1$. Define the constants

$$
\begin{aligned}
D\left(\beta, C_{-}, C_{+}, \beta_{0}, C_{0}, \nu\right)= & \sup _{C_{-} \leq x \leq C_{+}}\left[\frac { ( \zeta \beta ) ^ { - 1 + 1 / \zeta } } { 1 - x } \left\{\left(H\left(C_{0}\right)\left(\beta / \beta_{0}\right)^{1-1 / \zeta}\right)\right.\right. \\
& \left.\left.\vee\left(L_{\zeta}^{-1} \log \left(\left(\frac{2 \beta_{0}}{\beta}\right)^{1-1 / \zeta} \frac{2 H(x)}{\nu H\left(C_{0}\right)}\right)\right)\right\}\right]
\end{aligned}
$$

and

$$
\begin{aligned}
& M\left(\beta, C_{-}, C_{+}, \beta_{0}, C_{0}, \nu\right) \\
= & \exp \left\{\left(\zeta \beta_{0}\right)^{-1}\left(\left|\bar{D}^{-1}\left(C_{0}\right)\right|^{\zeta} \vee(\zeta-1)\right)\right\}
\end{aligned}
$$

$$
\vee \sup _{C_{-} \leq x \leq C_{+}} \exp \left\{(\zeta \beta)^{-1}\left(\frac{L_{\zeta}^{-1}}{x(1-\nu)} \log \left(\left(\frac{2 \beta_{0}}{\beta}\right)^{1-1 / \zeta} \frac{2 H(x)}{\nu H\left(C_{0}\right)}\right)\right)^{1 /(1-1 / \zeta)}\right\}
$$

imsart-aos ver. 2012/08/16 file: AOS1042_supp.tex date: August 22, 2012 
NEUVIAL, P. AND ROQUAIN, E.

for some $\nu \in(0,1)$ and by letting $H(x)=d\left(\bar{D}^{-1}(x)\right) / x$, for $x \in(0,1)$. Then (30) (in the main paper) holds for $M=M\left(\beta, C_{-}, C_{+}, \beta_{0}, C_{0}, \nu\right)$ and $D=D\left(\beta, C_{-}, C_{+}, \beta_{0}, C_{0}, \nu\right)$.

Proof. Corollary 4.3 entails that for any integer $m$ satisfying

$$
r_{m}^{l o c} \geq \frac{L_{\zeta}^{-1}}{C_{m}(1-\nu)}\left(\log \left(q_{m}^{o p t}\left(\beta_{0}, C_{0}\right) / q_{m}^{o p t}\right)-\log \nu\right),
$$

we have

$$
\begin{aligned}
& \frac{R_{m}\left(t_{m}^{\star}\right)-R_{m}\left(t_{m}^{B}\right)}{R_{m}\left(t_{m}^{B}\right)} \\
& \leq \frac{1}{1-C_{m}}\left\{\left(\frac{1}{q_{m}^{o p t}\left(\beta_{0}, C_{0}\right)}\right) \vee\left(L_{\zeta}^{-1} \frac{\log \left(q_{m}^{o p t}\left(\beta_{0}, C_{0}\right) / q_{m}^{o p t}\right)-\log \nu}{(\zeta \beta \log m)^{1-1 / \zeta}}\right)\right\} .
\end{aligned}
$$

From Lemma S-2.1 and the definition of $q_{m}^{o p t}$ given by (13), we have in the Subbotin location model (see Table S-1) that

$$
\begin{aligned}
\frac{r_{m}^{\text {loc }} C_{m}}{d\left(\bar{D}^{-1}\left(C_{m}\right)\right)} & \leq q_{m}^{\text {opt }} \\
\text { 21) } \quad q_{m}^{\text {opt }} & \leq \frac{r_{m}^{\text {loc }} C_{m}}{d\left(\bar{D}^{-1}\left(C_{m}\right)\right)}\left(1+\frac{\zeta-1}{\left(r_{m}^{l o c}\right)^{1 /(1-1 / \zeta)}}\right) \leq 2 \frac{r_{m}^{l o c} C_{m}}{d\left(\bar{D}^{-1}\left(C_{m}\right)\right)},
\end{aligned}
$$

where (S-21) holds by additionally assuming $\log m \geq(\zeta-1) /(\zeta \beta)$. Applying $(\mathrm{S}-20)$ and $(\mathrm{S}-21)$ (with $\left.\left(\beta, C_{m}\right)=\left(\beta_{0}, C_{0}\right)\right)$, we obtain that

$$
\begin{aligned}
\log \left(\frac{q_{m}^{\text {opt }}\left(\beta_{0}, C_{0}\right)}{q_{m}^{o p t}}\right)-\log \left(\frac{2 H\left(C_{m}\right)}{H\left(C_{0}\right)}\right) & \leq(1-1 / \zeta) \log \left(\frac{\zeta \beta_{0} \log m+\left|\bar{D}^{-1}\left(C_{0}\right)\right|^{\zeta}}{\zeta \beta \log m+\left|\bar{D}^{-1}\left(C_{m}\right)\right|^{\zeta}}\right) \\
& \leq(1-1 / \zeta) \log \left(\frac{2 \beta_{0}}{\beta}\right),
\end{aligned}
$$

whenever $\log m \geq\left|\bar{D}^{-1}\left(C_{0}\right)\right|^{\zeta} /\left(\zeta \beta_{0}\right)$. Therefore, (S-19) is satisfied for any integer $m$ satisfying the following inequalities:

$$
\begin{gathered}
\log m \geq\left(\zeta \beta_{0}\right)^{-1}\left(\left|\bar{D}^{-1}\left(C_{0}\right)\right|^{\zeta} \vee(\zeta-1)\right) \\
(\zeta \beta \log m)^{1-1 / \zeta} \geq \frac{L_{\zeta}^{-1}}{C_{m}(1-\nu)} \log \left(\left(\frac{2 \beta_{0}}{\beta}\right)^{1-1 / \zeta} \frac{2 H\left(C_{m}\right)}{\nu H\left(C_{0}\right)}\right) .
\end{gathered}
$$

imsart-aos ver. 2012/08/16 file: AOS1042_supp.tex date: August 22, 2012 
In particular, under $(\mathrm{BP})$ with some $0<C_{-} \leq C_{+}<1$, (S-19) holds for any $m \geq M\left(\beta, C_{-}, C_{+}, \beta_{0}, C_{0}, \nu\right)$. Furthermore, by using (S-20) (with $\left.\left(\beta, C_{m}\right)=\left(\beta_{0}, C_{0}\right)\right)$, we have

$$
1 / q_{m}^{o p t}\left(\beta_{0}, C_{0}\right) \leq \frac{H\left(C_{0}\right)}{\left(\zeta \beta_{0} \log m\right)^{1-1 / \zeta}}
$$

Thus, using (S-22) a second time, we have for any $m \geq M\left(\beta, C_{-}, C_{+}, \beta_{0}, C_{0}, \nu\right)$

$$
\begin{aligned}
& \quad R_{m}\left(t_{m}^{\star}\right)-R_{m}\left(t_{m}^{B}\right) \\
& R_{m}\left(t_{m}^{B}\right) /(\log m)^{1-1 / \zeta} \\
& \leq \frac{(\zeta \beta)^{-1+1 / \zeta}}{1-C_{m}}\left\{\left(H\left(C_{0}\right)\left(\beta / \beta_{0}\right)^{1-1 / \zeta}\right) \vee\left(L_{\zeta}^{-1} \log \left(\left(\frac{2 \beta_{0}}{\beta}\right)^{1-1 / \zeta} \frac{2 H\left(C_{m}\right)}{\nu H\left(C_{0}\right)}\right)\right)\right\} .
\end{aligned}
$$

This proves the result.

\section{S-3. Calculations for some standard densities.}

S-3.1. Equivalents for $\alpha_{m}^{o p t}\left(\beta_{0}, C_{0}\right)$. Let us recall that

$$
\alpha_{m}^{\text {opt }}\left(\beta_{0}, C_{0}\right)=\left(1+q_{m}^{\text {opt }}\left(\beta_{0}, C_{0}\right)\right)^{-1}
$$

is the choice we recommend for the use of BFDR/FDR thresholding, see (29). Proposition 4.1 provides an equivalent for $q_{m}^{o p t}\left(\beta_{0}, C_{0}\right)$. As a consequence, for large $m, \alpha_{m}^{o p t}\left(\beta_{0}, C_{0}\right)$ is equivalent to $\alpha_{m}^{\infty}\left(\beta_{0}, C_{0}\right)$, whose expression is given as follows:

- for the location model, $\zeta>1$

$$
\alpha_{m}^{\infty}\left(\beta_{0}, C_{0}\right)=\left\{1+\frac{C_{0}}{d\left(\bar{D}^{-1}\left(C_{0}\right)\right)}\left(\zeta \beta_{0} \log m\right)^{1-1 / \zeta}\right\}^{-1}
$$

- for the scale model, $\zeta \geq 1$,

$$
\alpha_{m}^{\infty}\left(\beta_{0}, C_{0}\right)=\left\{1+\frac{C_{0} / 2}{\bar{D}^{-1}\left(C_{0} / 2\right) d\left(\bar{D}^{-1}\left(C_{0} / 2\right)\right)} \zeta \beta_{0} \log m\right\}^{-1} .
$$

In particular, the cases $\zeta=1,2$ give rise to the following equivalents:

$$
\begin{array}{lc}
\alpha_{m}^{\infty}\left(\beta_{0}, C_{0}\right)=\left\{1+C_{0} e^{z_{0}^{2} / 2} \sqrt{4 \pi \beta_{0} \log m}\right\}^{-1} & \text { (Gaussian location); } \\
\alpha_{m}^{\infty}\left(\beta_{0}, C_{0}\right)=\left\{1+C_{0} \beta_{0} \sqrt{2 \pi} e^{\left(z_{0}^{\prime}\right)^{2} / 2}\left(z_{0}^{\prime}\right)^{-1} \log m\right\}^{-1} & \text { (Gaussian scale); } \\
\alpha_{m}^{\infty}\left(\beta_{0}, C_{0}\right)=\left\{1+\beta_{0}\left(\log \left(1 / C_{0}\right)\right)^{-1} \log m\right\}^{-1} & \text { (Laplace scale) }
\end{array}
$$

where $z_{0}$ and $z_{0}^{\prime}$ denote the quantiles of order $1-C_{0}$ and $1-C_{0} / 2$ of a standard Gaussian variable, respectively. 
S-3.2. Laplace scale model. In the Laplace scale model, it turns out that $\Psi_{m}^{-1}(\cdot)$ is an explicit function $\left(\Psi_{m}(t)=F_{m}(t) / t=t^{\sigma_{m}^{-1}-1}\right)$, so that we can investigate exact calculations for the risk of BFDR thresholding.

\section{S-3.2.1. Additional oracle inequalities and a lower bound.}

Proposition S-3.1. Consider the Laplace case $\phi(x)=x+\log 2$ and the corresponding scale model with parameters $\left(\tau_{m}, C_{m}\right) \in(1, \infty) \times(0,1)$. Let $\alpha_{m} \in(0,1 / 2)$ and $q_{m}=\alpha_{m}^{-1}-1$ be the corresponding recovery parameter.

(i) Let $g: x \in \mathbb{R} \mapsto e^{-x}+x-1 \in \mathbb{R}^{+}$. Then the BFDR threshold $t_{m}^{\star}$ at level $\alpha_{m}$ satisfies that for any $m \geq 2$,

$$
R_{m}\left(t_{m}^{\star}\right)-R_{m}\left(t_{m}^{B}\right)=C_{m} \pi_{1, m}\left(\frac{g\left(\log \left(q_{m} / \sigma_{m}\right)\right)}{\sigma_{m}}+\delta_{m}\right)
$$

for the remainder term $\delta_{m}=g\left(\frac{\log \left(q_{m} / \sigma_{m}\right)}{\sigma_{m}-1}\right)\left(q_{m}^{-1}-1\right)+\frac{\log \left(q_{m} / \sigma_{m}\right)}{\sigma_{m}-1}\left(\sigma_{m}^{-1}-\right.$ $\left.q_{m}^{-1}\right)$.

(ii) Let $\varepsilon \in(0,1), D_{1, m}=-\log \left(\pi_{0, m}(1-\varepsilon)\right)$ and $D_{2, m}=\log \left(m / \tau_{m}\right)$. Then the FDR threshold $\hat{t}_{m}^{F D R}$ at level $\alpha_{m}$ satisfies that for any $a \in\{1,2\}$, for any $m \geq 2$,

$$
\begin{aligned}
& R_{m}\left(\hat{t}_{m}^{F D R}\right)-R_{m}\left(t_{m}^{B}\right) \\
\leq & \pi_{1, m}\left(\frac{\alpha_{m}}{1-\alpha_{m}}+C_{m} \frac{\left(\log \left(\alpha_{m}^{-1} / \sigma_{m}\right)+D_{a, m}\right)_{+}}{\sigma_{m}-1}\right)+\frac{\alpha_{m} / m}{\left(1-\alpha_{m}\right)^{2}} \\
(\mathrm{~S}-24) & \\
\quad+ & \pi_{1, m} \mathbf{1}\{a=1\} \exp \left\{-\frac{m \varepsilon^{2} C_{m}}{4\left(\tau_{m}+1\right)} \frac{\left(1-\left(\log \left(\alpha_{m}^{-1} / \sigma_{m}\right)+D_{1, m}\right)_{+}\right)_{+}}{\sigma_{m}-1}\right\} .
\end{aligned}
$$

Proposition S-3.1 is proved in Section S-3.2.2. Expression (S-23) results from direct calculations while inequality (S-24) relies on Theorem 3.2. As we consider the Laplace scale model, we can easily check that the optimal recovery parameter is $\sigma_{m}$, that is, we have $\Psi_{m}^{-1}\left(\sigma_{m} \tau_{m}\right)=t_{m}^{B}$. Expression (S-23) gives the excess risk when choosing $q_{m}$ instead of $\sigma_{m}$ as recovery parameter in the BFDR threshold, which is proved to strongly depend on the behavior of $g(\cdot)$. Next, inequality (S-24) can be seen as an improvement over (27) in the special case of a Laplace scale model: while $K_{m} / r_{m}^{s c}$ is of the same order as $C_{m} / \sigma_{m}$ in that case (because $K_{m}=C_{m} \log \left(1 / C_{m}\right)$ and because we have $\sigma_{m} \sim \log \tau_{m} /\left(\log \left(1 / C_{m}\right)\right)$ by (S-28)), the remainder terms are of smaller order in (S-24) and inequality (S-24) is true for any $m \geq 2$.

Proposition S-3.1 entails the following result. 
Corollary S-3.2. Consider the Laplace scale model satisfying assumption $(\mathrm{BP})$ and $(\mathrm{Sp})$. Then for any $\alpha_{m} \in(0,1)$ with recovery parameter $q_{m}=\alpha_{m}^{-1}-1$, we have

$$
R_{m}\left(t_{m}^{\star}\left(\alpha_{m}\right)\right)-R_{m}\left(t_{m}^{B}\right)=o\left(R_{m}\left(t_{m}^{B}\right) /\left(\log \tau_{m}\right)\right) \text { if and only if } q_{m} \sim \sigma_{m} .
$$

Furthermore, by considering a sequence of parameter pairs $\left(\tau_{m}, C_{m}\right) \in(1, \infty) \times$ $(0,1), m \geq 2$, with $\left(C_{m}\right)_{m}$ satisfying $(\mathrm{BP})$ and $\tau_{m}=m^{\beta}, \beta \in \mathcal{B}$, for some subset $\mathcal{B}$ of $(0,1]$ containing more than two elements, the two following statements hold:

(i) for any sequence $\left(\alpha_{m}\right)_{m}$ with $\alpha_{m} \in(0,1)$ (that does not depend of $\beta$ ),

$$
\liminf _{m}\left\{(\log m) \sup _{\beta \in \mathcal{B}}\left(\frac{R_{m}\left(t_{m}^{\star}\left(\alpha_{m}\right)\right)-R_{m}\left(t_{m}^{B}\right)}{R_{m}\left(t_{m}^{B}\right)}\right)\right\}>0
$$

(ii) additionally assuming $\mathcal{B}=\left[\beta_{-}, 1\right]$ for some $\beta_{-} \in(0,1)$ and taking $\alpha_{m} \propto 1 /(\log m)$,

$$
\limsup _{m}\left\{(\log m) \sup _{\beta \in \mathcal{B}}\left(\frac{R_{m}\left(t_{m}^{\star}\left(\alpha_{m}\right)\right)-R_{m}\left(t_{m}^{B}\right)}{R_{m}\left(t_{m}^{B}\right)}\right)\right\}<\infty .
$$

Corollary S-3.2 is proved in Section S-3.2.3. Importantly, in (S-26) and (S-27), it should be noted that the sequence $\left(C_{m}\right)_{m}$ does not change when changing $\beta$ : the parameters $\tau_{m}$ and $C_{m}$ are implicitly considered as the "generative" parameters of the model and thus are taken independently. A consequence of Corollary S-3.2 (i) (ii) is that the rate $\rho_{m}=1 /(\log m)$ obtained in Corollary 4.4 (iii) can not be improved for BFDR thresholding in the particular case of a Laplace scale model and over a sparsity range $\beta \in\left[\beta_{-}, 1\right]$ for some $\beta_{-} \in(0,1)$.

S-3.2.2. Proof of Proposition S-3.1. In the Laplace case, some useful relations are reported in Table S-2. Also, recall that from Lemma S-2.2, we have

$$
\log \tau_{m}+\log \sigma_{m}=\left(\sigma_{m}-1\right) \log \left(1 / C_{m}\right),
$$

that is, $\tau_{m} \sigma_{m}=C_{m}^{1-\sigma_{m}}$. Furthermore, under (BP) and (Sp), we have $\log \tau_{m} \sim$ $\log \left(1 / C_{m}\right) \sigma_{m}$.

imsart-aos ver. 2012/08/16 file: AOS1042_supp.tex date: August 22, 2012 


\begin{tabular}{|c|c||c|c|}
\hline$\phi(x)$ & $x+\log 2$ & $F_{m}(t)$ & $t_{m}^{\sigma_{m}^{-1}}$ \\
$d(x)$ & $e^{-x} / 2$ & $\Psi_{m}(t)$ & $t^{\sigma_{m}^{-1}-1}$ \\
$\bar{D}(x)$ & $e^{-x} / 2$ & $\Psi_{m}^{-1}(v)$ & $v^{1 /\left(\sigma_{m}^{-1}-1\right)}$ \\
$\bar{D}^{-1}(u)$ & $-\log (2 u)$ & $F_{m}\left(\Psi_{m}^{-1}(v)\right)$ & $(1 / v)^{1 /\left(\sigma_{m}-1\right)}$ \\
\hline
\end{tabular}

TABLE S-2

Some calculations for the Laplace scale model. $x \geq 0 ; t \in(0,1) ; v>0 ; u \leq 1 / 2$.

Let us start by proving (S-23). By definition, we have $R_{m}\left(t_{m}^{\star}\right)-R_{m}\left(t_{m}^{B}\right)=$ $C_{m} \pi_{1, m}\left(Z_{1, m}+Z_{2, m}\right)$, where

$$
\begin{aligned}
& \left.Z_{1, m}=\tau_{m} C_{m}^{-1}\left(\Psi_{m}^{-1}\left(q_{m} \tau_{m}\right)\right)-t_{m}^{B}\right) \\
& Z_{2, m}=1-C_{m}^{-1} F_{m}\left(\Psi_{m}^{-1}\left(q_{m} \tau_{m}\right)\right) .
\end{aligned}
$$

On the one hand, since $t_{m}^{B}=\left(C_{m}\right)^{\sigma_{m}}$ and using (S-28) twice, we get

$$
\begin{aligned}
Z_{1, m} & =\tau_{m} C_{m}^{-1+\sigma_{m}}\left(\left(C_{m}\right)^{-\sigma_{m}} \exp \left(-\frac{\log \left(q_{m} \tau_{m}\right)}{1-\sigma_{m}^{-1}}\right)-1\right) \\
& =\sigma_{m}^{-1}\left(\exp \left(-\frac{\log q_{m}+\log \tau_{m}+\left(\sigma_{m}-1\right) \log C_{m}}{1-\sigma_{m}^{-1}}\right)-1\right) \\
& =\sigma_{m}^{-1}\left(\exp \left(-\frac{\log q_{m}-\log \sigma_{m}}{1-\sigma_{m}^{-1}}\right)-1\right) .
\end{aligned}
$$

On the other hand, by using again (S-28), we obtain

$$
\begin{aligned}
Z_{2, m} & =1-\exp \left(-\frac{\log q_{m}+\log \tau_{m}+\left(\sigma_{m}-1\right) \log C_{m}}{\sigma_{m}-1}\right) \\
& =1-\exp \left(-\frac{\log q_{m}-\log \sigma_{m}}{\sigma_{m}-1}\right) .
\end{aligned}
$$

This implies, by denoting $\kappa_{m}=\log q_{m}-\log \sigma_{m}$ and by using the function $g$,

$$
\begin{aligned}
& \left(C_{m} \pi_{1, m}\right)^{-1}\left(R_{m}\left(t_{m}^{\star}\right)-R_{m}\left(t_{m}^{B}\right)\right) \\
& =\sigma_{m}^{-1}\left(-1+e^{-\kappa_{m}}\left(1-\frac{\kappa_{m}}{\sigma_{m}-1}+g\left(\frac{\kappa_{m}}{\sigma_{m}-1}\right)\right)\right)+\frac{\kappa_{m}}{\sigma_{m}-1}-g\left(\frac{\kappa_{m}}{\sigma_{m}-1}\right) .
\end{aligned}
$$

This leads to (S-23), because $e^{-\kappa_{m}}=\sigma_{m} / q_{m}$.

Next, we can prove (S-24) by applying Theorem 3.2. By using the above computation of $Z_{2, m}$, we have

$$
\begin{aligned}
\gamma_{m}^{\varepsilon} & \leq C_{m}\left(1-\exp \left(-\frac{\log \left(\alpha_{m}^{-1} / \sigma_{m}\right)-\log \left(\pi_{0, m}(1-\varepsilon)\right)}{\sigma_{m}-1}\right)\right)_{+} \\
& \leq C_{m} \frac{\left(\log \left(q_{m} / \sigma_{m}\right)-\log \left(\pi_{0, m}(1-\varepsilon)\right)\right)_{+}}{\sigma_{m}-1}
\end{aligned}
$$

imsart-aos ver. 2012/08/16 file: AOS1042_supp.tex date: August 22, 2012 
because for any $u \in \mathbb{R},\left(1-e^{-u}\right)_{+} \leq u_{+}$. This gives (S-24) for $a=1$. The case where $a=2$ is similar:

$$
\begin{aligned}
\left(1-C_{m}^{-1} F_{m}(\alpha / m)\right)_{+} & \leq\left(1-\exp \left(-\frac{\log \left(\alpha_{m}^{-1} m\right)+\left(\sigma_{m}-1\right) \log C_{m}}{\sigma_{m}-1}\right)\right)_{+} \\
& \leq \frac{\left(\log \left(\alpha_{m}^{-1} / \sigma_{m}\right)+\log \left(m / \tau_{m}\right)\right)_{+}}{\sigma_{m}-1}
\end{aligned}
$$

by using (S-28). This finishes the proof of Proposition S-3.1.

S-3.2.3. Proof of Corollary S-3.2. Assume (BP) and (Sp) and let us prove the equivalence (S-25). First, we can assume that condition " $q_{m} \rightarrow \infty$ and $\log q_{m}=o\left(\sigma_{m}\right)$ " is satisfied: otherwise, both assertions in (S-25) are false by using Corollary S-1.1 (i) and because $\sigma_{m} \sim\left(\log \left(1 / C_{m}\right)\right)^{-1} \log \tau_{m}$ (by using (S-28)). Now, assume that $\log \left(q_{m} / \sigma_{m}\right)$ has a limit in $\mathbb{R} \cup\{-\infty,+\infty\}$. As $g$ satisfies $g(x)=O\left(x^{2}\right)$ as $x \rightarrow 0 ; g(x) \sim x$ as $x \rightarrow+\infty ; g(\log u) \sim 1 / u$ as $u \rightarrow 0$, we easily check from (S-23) that the following holds:

- if $\log \left(q_{m} / \sigma_{m}\right) \rightarrow 0$, the relative excess risk tends to zero faster than $1 /\left(\log \tau_{m}\right)$

- if $\log \left(q_{m} / \sigma_{m}\right) \rightarrow l \in \mathbb{R} \backslash\{0\}$, the relative excess risk is of order $1 /\left(\log \tau_{m}\right)$;

- if $\log \left(q_{m} / \sigma_{m}\right) \rightarrow-\infty$ or $\log \left(q_{m} / \sigma_{m}\right) \rightarrow+\infty$, the relative excess risk tends to zero slower than $1 /\left(\log \tau_{m}\right)$;

Let us now prove that (S-25) holds: if $q_{m} \sim \sigma_{m}$, then $\log \left(q_{m} / \sigma_{m}\right) \rightarrow 0$ and what is above proves that the LHS of (S-25) is true. Conversely, if $q_{m} / \sigma_{m}$ does not converge to 1 , then $\log \left(q_{m} / \sigma_{m}\right)$ converges to some non-zero element (possibly infinite), by considering a subsequence. From what is above, this implies that one subsequence of the relative excess risk is of order at least $1 /\left(\log \tau_{m}\right)$ and thus that the LHS of (S-25) is false.

Next, let us prove (S-26). Recall that $\sigma_{m} \sim \beta \log m /\left(\log \left(1 / C_{m}\right)\right)$. Hence, if the limit in (S-26) is zero, we have from (S-25) that for any $\beta \in \mathcal{B}$, $q_{m} \sim\left(\log \left(1 / C_{m}\right)\right)^{-1} \beta \log m$ and thus $q_{m}\left(\log \left(1 / C_{m}\right)\right) /(\log m) \rightarrow \beta$. This is impossible as soon as $\mathcal{B}$ contains more than two elements, because $q_{m}$ and $C_{m}$ do not depend of $\beta$. This proves that the limit in (S-26) is positive and establishes (S-26).

Finally, (S-27) is an easy consequence of (S-23), by using that $\sigma_{m} \geq$ $\beta \log m /\left(\log \left(1 / C_{m}\right)\right)$ and thus

$\sup _{\beta_{-} \leq \beta \leq 1}\left\{\frac{\log \left(q_{m} / \sigma_{m}\right)}{\sigma_{m}}\right\} \leq \log \left(1 / C_{m}\right) \frac{\log \left(q_{m} /(\log m)\right)-\log \beta_{-}+\log \log \left(1 / C_{m}\right)}{\beta_{-} \log m}$.

imsart-aos ver. 2012/08/16 file: AOS1042_supp.tex date: August 22, 2012 
S-3.3. Laplace location model. Our results do not cover the case of the Laplace location model because $\phi(u)=u+\log 2$ is not strictly convex and thus $f_{m}$ is only non-increasing, not decreasing. In this case, while the optimal classification procedures are still thresholding procedures, the Bayes threshold is 0 or 1 whenever $\tau_{m} \leq e^{-\mu_{m}}$ or $\tau_{m} \geq e^{\mu_{m}}$, respectively. This can be derived from the exact expression of $F_{m}$ provided in Proposition 25 of [1] (item 3). Nevertheless, the Bayes threshold is still unique in $(0,1)$ as soon as the parameters $\left(\tau_{m}, \mu_{m}\right)$ satisfy the constraint

$$
e^{-\mu_{m}}<\tau_{m}<e^{\mu_{m}}
$$

Moreover, this entails $1 / 2<C_{m}<1-e^{-\mu_{m}} / 2, q_{m}^{o p t}=C_{m} /\left(1-C_{m}\right)$ and $R_{m}\left(t_{m}^{B}\right)=2 \pi_{1, m}\left(1-C_{m}\right)$. In particular, one major difference with the cases considered in the main paper [2] is that $q_{m}^{\text {opt }}$ does not tend to infinity under (BP) and (Sp). Also, we have $r_{m}^{l o c}=1$ as defined in (21). Under Assumption (S-30), Theorems 3.1 and 3.2 can be readily applied to obtain upper bounds for the excess risk of BFDR/FDR thresholding. While this proves that BFDR thresholding is still asymptotically optimal when choosing $q_{m}-q_{m}^{o p t}=o(1)$, we cannot derive such a statement directly for FDR thresholding. This comes from the fact that we used a "one-sided" concentration argument while bounding the type I error. Rather, we would need a "two-sided" concentration argument, which seems feasible but possibly technical.

We have also performed numerical experiments for the Laplace location model, see Figure S-3. These experiments show that this model is somewhat singular: while the adaptation w.r.t. $\beta$ is stronger than for the other models (the relative excess risk is even independent of $\beta$ for BFDR thresholding), the sensitivity to the mis-specification of $C_{m}$ is much higher. This behavior is in agreement with the expression of $q_{m}^{o p t}$ which involves $C_{m}$ but not $\beta$.

S-3.4. Gaussian models. Let us consider the special case where $d(\cdot)$ is the standard Gaussian density. In that case, while $\Psi_{m}$ is not easily invertible, an explicit expression can be derived for $f_{m}^{-1}$, see Table S-3. By using (20) in Remark 3.3, Theorems 3.1 and 3.2 lead to explicit upper bounds for the excess risk of BFDR/FDR thresholding. In contrast with the bounds derived in Section 4.2, they are valid for any $m \geq 2$, but the quantity " $\log \left(q_{m} / q_{m}^{o p t}\right)$ " is replaced by " $\log q_{m}$ " (up to constant terms). The reason for this is that

$$
\gamma_{m}=\left(F_{m}\left(\Psi_{m}^{-1}\left(q_{m}^{o p t} \tau_{m}\right)\right)-F_{m}\left(\Psi_{m}^{-1}\left(q_{m} \tau_{m}\right)\right)\right)_{+}
$$

involves a variation of $q_{m}$ around $q_{m}^{o p t}$, while

$$
C_{m}-F_{m}\left(f_{m}^{-1}\left(q_{m} \tau_{m}\right)\right)=F_{m}\left(f_{m}^{-1}\left(\tau_{m}\right)\right)-F_{m}\left(f_{m}^{-1}\left(q_{m} \tau_{m}\right)\right)
$$


involves a variation of $q_{m}$ around 1 . When choosing $q_{m} \propto q_{m}^{o p t}$, this method inflates the upper-bound by a factor $\log \log \tau_{m}$ w.r.t. the bounds derived in Section 4.2.

\begin{tabular}{|c|c|c|}
\hline & Gaussian location & Gaussian scale \\
\hline Parameter & $\mu_{m}=-\bar{\Phi}^{-1}\left(C_{m}\right)$ & $\log \tau_{m}+\log \sigma_{m}$ \\
\hline & $+\sqrt{\left(\bar{\Phi}^{-1}\left(C_{m}\right)\right)^{2}+2 \log \tau_{m}}$ & $=\left(\bar{\Phi}^{-1}\left(C_{m} / 2\right)\right)^{2}\left(\sigma_{m}^{2}-1\right) / 2$ \\
\hline$F_{m}(t)$ & $\bar{\Phi}\left(\bar{\Phi}^{-1}(t)-\mu_{m}\right)$ & $2 \bar{\Phi}\left(\bar{\Phi}^{-1}(t / 2) / \sigma_{m}\right)$ \\
\hline$f_{m}(t)$ & $\exp \left(\mu_{m}\left(\bar{\Phi}^{-1}(t)-\mu_{m} / 2\right)\right)$ & $\sigma_{m}^{-1} \exp \left\{\left(1-\sigma_{m}^{-2}\right)\left(\bar{\Phi}^{-1}(t / 2)\right)^{2} / 2\right\}$ \\
\hline$f_{m}^{-1}(u)$ & $\bar{\Phi}\left((\log u) / \mu_{m}+\mu_{m} / 2\right)$ & $2 \bar{\Phi}\left(\left(2\left(\log \left(\sigma_{m} u\right)\right) \sigma_{m}^{2} /\left(\sigma_{m}^{2}-1\right)\right)^{1 / 2}\right)$ \\
\hline$F_{m}\left(f_{m}^{-1}\left(q_{m} \tau_{m}\right)\right)$ & $\bar{\Phi}\left(\left(\log q_{m}\right) / \mu_{m}+\bar{\Phi}^{-1}\left(C_{m}\right)\right)$ & $2 \bar{\Phi}\left(\left(\left(\bar{\Phi}^{-1}\left(C_{m} / 2\right)\right)^{2}+\frac{2 \log q_{m}}{\sigma_{m}^{2}-1}\right)^{1 / 2}\right)$ \\
\hline
\end{tabular}

TABLE S-3

Some calculations for the Gaussian location and scale models. $\bar{\Phi}(x)=\mathbb{P}(Z \geq x)$ for $Z \sim \mathcal{N}(0,1) ; t \in(0,1) ; u>0$

S-4. Study of the weighted mis-classification risk. According to Section 6.2 in [2], consider the weighted mis-classification risk:

$$
R_{m, \lambda_{m}}\left(\hat{t}_{m}\right)=\mathbb{E}\left(\pi_{0, m} \hat{t}_{m}+\lambda_{m} \pi_{1, m}\left(1-F_{m}\left(\hat{t}_{m}\right)\right)\right),
$$

for an additional known factor $\lambda_{m} \in\left(1, \tau_{m}\right)$. The methodology proposed in the main paper [2] can be readily extended to this risk by following the proof of [2]. The corresponding results are given below. First, assuming $\left(A\left(F_{m}, \tau_{m} / \lambda_{m}\right)\right)$, the Bayes threshold is given by $t_{m}^{B}=f_{m}^{-1}\left(\tau_{m} / \lambda_{m}\right)$. Condition (BP) remains unchanged while (Sp) becomes

$$
\tau_{m} \text { and } \tau_{m} / \lambda_{m} \text { tends to infinity as } m \rightarrow \infty .
$$

For BFDR thresholding, the optimal recovery parameter is given by

$$
q_{m}^{o p t}=\tau_{m}^{-1} \Psi_{m}\left(f_{m}^{-1}\left(\tau_{m} / \lambda_{m}\right)\right)=\frac{C_{m}}{\tau_{m} t_{m}^{B}},
$$

Next, we can prove the following results.

TheOREM S-4.1. Assume $\left(\mathrm{A}\left(F_{m}, \tau_{m}\right)\right)$ and consider the BFDR threshold $t_{m}^{\star}$ at a level $\alpha_{m} \in\left(\left(1+f_{m}\left(0^{+}\right) / \tau_{m}\right)^{-1}, \pi_{0, m}\right)$ corresponding to a recovery parameter $q_{m}=\alpha_{m}^{-1}-1$. Consider $q_{m}^{o p t} \geq 1$ the optimal recovery parameter given by (S-32). Then the following holds:

(i) if $q_{m} \lambda_{m} \geq 1$, we have for any $m \geq 2$,

$$
\begin{aligned}
& R_{m, \lambda_{m}}\left(t_{m}^{\star}\right)-R_{m, \lambda_{m}}\left(t_{m}^{B}\right) \leq \pi_{1, m} \lambda_{m}\left\{\left(C_{m} /\left(q_{m} \lambda_{m}\right)-C_{m} /\left(q_{m}^{o p t} \lambda_{m}\right)\right) \vee \gamma_{m}\right\}, \\
& \text { imsart-aos ver. } 2012 / 08 / 16 \text { file: A0S1042_supp.tex date: August 22, } 2012
\end{aligned}
$$


where we let $\gamma_{m}=\left(C_{m}-F_{m}\left(\Psi_{m}^{-1}\left(q_{m} \tau_{m}\right)\right)_{+}\right.$. In particular, under (BP), if $q_{m} \lambda_{m} \rightarrow+\infty$ and $\gamma_{m} \rightarrow 0$, the BFDR threshold $t_{m}^{\star}$ is asymptotically optimal at rate $\left(q_{m} \lambda_{m}\right)^{-1}+\gamma_{m}$.

(ii) we have for any $m \geq 2$,

$$
\frac{R_{m, \lambda_{m}}\left(t_{m}^{\star}\right)}{R_{m, \lambda_{m}}\left(t_{m}^{B}\right)} \geq \frac{\pi_{1, m} \lambda_{m}}{R_{m, \lambda_{m}}\left(t_{m}^{B}\right)}\left(1-\left(1-\left(q_{m} \lambda_{m}\right)^{-1}\right)_{+} F_{m}\left(q_{m}^{-1} \tau_{m}^{-1}\right)\right) .
$$

In particular, under $(\mathrm{BP})$, if $R_{m, \lambda_{m}}\left(t_{m}^{B}\right) \sim \pi_{1, m} \lambda_{m}\left(1-C_{m}\right)$ and if

$$
\liminf _{m}\left\{\frac{1-\left(1-\left(q_{m} \lambda_{m}\right)^{-1}\right)_{+} F_{m}\left(q_{m}^{-1} \tau_{m}^{-1}\right)}{1-C_{m}}\right\}>1
$$

$t_{m}^{\star}$ is not asymptotically optimal.

Theorem S-4.2. Let $\varepsilon \in(0,1)$, assume $\left(\mathrm{A}\left(F_{m}, \tau_{m}\right)\right)$ and consider the FDR threshold $\hat{t}_{m}^{F D R}$ at level $\alpha_{m}>(1-\varepsilon)^{-1}\left(\pi_{0, m}+\pi_{1, m} f_{m}\left(0^{+}\right)\right)^{-1}$. Then the following holds: for any $m \geq 2$,

$$
R_{m, \lambda_{m}}\left(\hat{t}_{m}^{F D R}\right)-R_{m, \lambda_{m}}\left(t_{m}^{B}\right) \leq \pi_{1, m} \frac{\alpha_{m}}{1-\alpha_{m}}+m^{-1} \frac{\alpha_{m}}{\left(1-\alpha_{m}\right)^{2}}
$$

for $\gamma_{m}^{\varepsilon}=\left(C_{m}-F_{m}\left(\Psi_{m}^{-1}\left(q_{m}^{\varepsilon} \tau_{m}\right)\right)\right)_{+}$with $q_{m}^{\varepsilon}=\left(\alpha_{m} \pi_{0, m}(1-\varepsilon)\right)^{-1}-1$ and $\gamma_{m}^{\prime}=\left(C_{m}-F_{m}\left(\alpha_{m} / m\right)\right)_{+}$. In particular, under (BP) and assuming $\lambda_{m} q_{m} \rightarrow$ $\infty$ and $q_{m}^{-1}=O(1)$,

(i) if $\tau_{m} / m=O(1), \gamma_{m}^{\varepsilon} \rightarrow 0$ and $\forall \kappa>0, e^{-\kappa m / \tau_{m}}=o\left(\gamma_{m}^{\varepsilon}\right)$, the FDR threshold $\hat{t}_{m}^{F D R}$ is asymptotically optimal at rate $\left(q_{m} \lambda_{m}\right)^{-1}+\gamma_{m}^{\varepsilon}$.

(ii) if $m / \tau_{m} \rightarrow l \in(0,+\infty)$ with $\gamma_{m}^{\prime} \rightarrow 0$, the FDR threshold $\hat{t}_{m}^{F D R}$ is asymptotically optimal at rate $\left(q_{m} \lambda_{m}\right)^{-1}+\gamma_{m}^{\prime}$.

The rates in the location and scale models should be modified as follows:

$$
\begin{aligned}
r_{m}^{l o c} & =\phi^{\prime} \circ \phi^{-1}\left(\log \left(\tau_{m} / \lambda_{m}\right)+\phi\left(\left|\bar{D}^{-1}\left(C_{m}\right)\right|\right)\right) \\
r_{m}^{s c} & =\left(\operatorname{Id} \times \phi^{\prime}\right) \circ \phi^{-1}\left(\log \left(\tau_{m} / \lambda_{m}\right)+\phi\left(\bar{D}^{-1}\left(C_{m} / 2\right)\right)\right),
\end{aligned}
$$

which gives in the $\zeta$-Subbotin case $r_{m}^{l o c}=\left(\zeta \log \left(\tau_{m} / \lambda_{m}\right)+\left|\bar{D}^{-1}\left(C_{m}\right)\right|^{\zeta}\right)^{1-1 / \zeta}$ and $r_{m}^{s c}=\zeta \log \left(\tau_{m} / \lambda_{m}\right)+\left(\bar{D}^{-1}\left(C_{m} / 2\right)\right)^{\zeta}$. We easily prove the following proposition.

imsart-aos ver. 2012/08/16 file: AOS1042_supp.tex date: August 22, 2012 
Proposition S-4.3. Consider $d(x)=e^{-\phi(|x|)}$ for a function $\phi$ satisfying $(\mathrm{A}(\phi))$ in the scale model or $\left(\mathrm{A}^{\prime}(\phi)\right)$ in the location model. Let $\left(\tau_{m}, C_{m}\right) \in$ $(1, \infty) \times(0,1)$ be the parameters of the model. Let $r_{m}$ being equal to $r_{m}^{\text {loc }}$ defined by (S-37) in the location model and to $r_{m}^{s c}$ defined by (S-38) in the scale model. Then, under (BP) and (S-Sp), we have

$$
\begin{aligned}
R_{m, \lambda_{m}}\left(t_{m}^{B}\right) & \sim \pi_{1, m} \lambda_{m}\left(1-C_{m}\right) . \\
t_{m}^{B} & =O\left(R_{m, \lambda_{m}}\left(t_{m}^{B}\right) / r_{m}\right)
\end{aligned}
$$

Furthermore, for a $\zeta$-Subbotin density,

$$
\lambda_{m} q_{m}^{\text {opt }} \sim \begin{cases}\frac{C_{m}}{d\left(\bar{D}^{-1}\left(C_{m}\right)\right)}\left(\zeta \log \left(\tau_{m} / \lambda_{m}\right)\right)^{1-1 / \zeta} & \text { for location, } \zeta>1 \\ \frac{C_{m}^{-1}\left(C_{m} / 2\right) d\left(\bar{D}^{-1}\left(C_{m} / 2\right)\right)}{\bar{D}^{-1}} \log \left(\tau_{m} / \lambda_{m}\right) & \text { for scale, } \zeta \geq 1\end{cases}
$$

Theorems S-4.1 and S-4.2 may be applied to the location and scale models to provide an explicit convergence rate for the risk $R_{m, \lambda_{m}}$, as stated below.

COROllary S-4.4. Consider $d(x)=e^{-\phi(|x|)}$ for a function $\phi$ satisfying $(\mathrm{A}(\phi))$ in the scale model or $\left(\mathrm{A}^{\prime}(\phi)\right)$ in the location model. Let $\left(\tau_{m}, C_{m}\right) \in$ $(1, \infty) \times(0,1)$ be the parameters of the model. Let $r_{m}$ and $\psi(\cdot)$ be defined as follows:

- in the location model, $r_{m}=r_{m}^{l o c}$ defined by (S-37) and $\psi=\phi^{\prime} \circ \phi^{-1}$;

- in the scale model, $r_{m}=r_{m}^{s c}$ defined by $(\mathrm{S}-38)$ and $\psi=\left(I d \times \phi^{\prime}\right) \circ \phi^{-1}$.

Assume (BP) and (S-Sp). Consider the BFDR threshold $t_{m}^{\star}$ at a level $\alpha_{m} \in$ $(0,1)$, with a corresponding recovery parameter $q_{m}=\alpha_{m}^{-1}-1$ satisfying $q_{m}^{-1}=O(1)$. Then the following holds:

(i) the BFDR threshold $t_{m}^{\star}$ is asymptotically optimal if

$$
q_{m} \lambda_{m} \rightarrow \infty \text { and } \log \left(q_{m} \lambda_{m}\right)=o\left(r_{m}\right)
$$

in which case it is asymptotically optimal at rate $\rho_{m}=\left(q_{m} \lambda_{m}\right)^{-1}+$ $\left(\log \left(q_{m} \lambda_{m} / r_{m}\right)\right)_{+} / r_{m}$. Additionally, if $\phi$ satisfies $(B(\phi))$ and $\psi$ satisfies $(C(\psi))$, the BFDR threshold $t_{m}^{\star}$ is asymptotically optimal if and only if (S-42) holds.

(ii) Assume moreover that there exists $\lambda>0$ such that $\psi(x)=O\left(e^{\lambda x}\right)$ for $x \rightarrow+\infty$ and that the sparsity regime $\tau_{m}$ satisfies

$$
m / \tau_{m} \geq\left(\log \tau_{m}\right)^{1+\theta} \text { for some } \theta>0 ; \quad \text { or } \quad m / \tau_{m} \rightarrow l \in(0,+\infty) .
$$

imsart-aos ver. 2012/08/16 file: AOS1042_supp.tex date: August 22, 2012 
Then, the FDR threshold $\hat{t}_{m}^{F D R}$ at a level $\alpha_{m}$ satisfying (S-42) is asymptotically optimal at rate $\rho_{m}=\left(q_{m} \lambda_{m}\right)^{-1}+\left(\log \left(q_{m} \lambda_{m} / r_{m}\right)\right)_{+} / r_{m}$.

Corollary S-4.4 is illustrated in Table S-4.

\begin{tabular}{|c|c|c|}
\hline Model & $\zeta$-Subbotin location, $\zeta>1$ & $\zeta$-Subbotin scale, $\zeta \geq 1$ \\
\hline $\begin{array}{c}\text { Parameter } \\
r_{m} \text { in }(\mathrm{S}-37) \text { or }(\mathrm{S}-38)\end{array}$ & $\begin{array}{l}\mu_{m} \sim(\zeta \beta \log m)^{1 / \zeta} \\
r_{m}^{l o c} \sim(\zeta \beta \log m)^{1-1 / \zeta}\end{array}$ & $\begin{aligned} \sigma_{m} & \sim \frac{(\zeta \beta \log m)^{1 / \zeta}}{\bar{D}^{-1}\left(C_{m} / 2\right)} \\
r_{m}^{s c} & \sim \zeta \beta \log m\end{aligned}$ \\
\hline \multicolumn{3}{|l|}{ "Bayes' threshold } \\
\hline $\begin{array}{c}m^{\beta} \lambda_{m}^{-1} t_{m}^{B} \\
R_{m, \lambda_{m}}\left(t_{m}^{B}\right) \\
\lambda_{m} q_{m}^{o p t} \text { in }(\mathrm{S}-32)\end{array}$ & $\begin{array}{l}\sim \frac{d\left(\bar{D}^{-1}\left(C_{m}\right)\right)}{(\zeta \beta \log m)^{1-1 / \zeta}} \\
\sim m^{-\beta} \lambda_{m}\left(1-C_{m}\right) \\
\sim \frac{C_{m}(\zeta \beta \log m)^{1-1 / \zeta}}{d\left(\bar{D}^{-1}\left(C_{m}\right)\right)}\end{array}$ & $\begin{array}{l}\sim \frac{2 \bar{D}^{-1}\left(C_{m} / 2\right) d\left(\bar{D}^{-1}\left(C_{m} / 2\right)\right)}{\zeta \beta \log m} \\
\sim m^{-\beta} \lambda_{m}\left(1-C_{m}\right) \\
\sim \frac{C_{m} \zeta \beta \log m}{2 \bar{D}^{-1}\left(C_{m} / 2\right) d\left(\bar{D}^{-1}\left(C_{m} / 2\right)\right)}\end{array}$ \\
\hline \multicolumn{3}{|l|}{ (B)FDR threshold } \\
\hline $\begin{array}{c}\text { Optimality } \\
\text { condition }(\mathrm{S}-42)\end{array}$ & $\begin{array}{l}q_{m} \lambda_{m} \rightarrow \infty \\
\log \left(q_{m} \lambda_{m}\right)=o\left((\log m)^{1-1 / \zeta}\right)\end{array}$ & $\begin{array}{l}q_{m} \lambda_{m} \rightarrow \infty \\
\log \left(q_{m} \lambda_{m}\right)=o(\log m)\end{array}$ \\
\hline $\begin{array}{c}\text { Rate } \rho_{m} \\
\text { for } q_{m} \propto q_{m}^{o p t}\end{array}$ & $1 /(\log m)^{1-1 / \zeta}$ & $1 /(\log m)$ \\
\hline
\end{tabular}

TABLE S-4

Summary of our results for the $\lambda_{m}$-weighted mis-classification risk. $\zeta$-Subbotin density in the sparsity regime $\tau_{m}=m^{\beta}$, $0<\beta \leq 1$, under $(\mathrm{BP})$, for a $\lambda_{m}$ such that $\log \lambda_{m}=o\left(\log \tau_{m}\right)$ and for $q_{m}^{-1}=O(1)$.

\section{S-5. Expressions for tails and quantiles.}

Lemma S-5.1. Let $d(x)=e^{-\phi(|x|)}$ for any $x \in \mathbb{R}$, where $\phi$ is a function satisfying $(\mathrm{A}(\phi))$. Then $\bar{D}(x)=\int_{x}^{+\infty} e^{-\phi(|u|)} d u$ has the following properties:

- for any $x>0$, we have

$$
\bar{D}(x) \leq d(x) / \phi^{\prime}(x)
$$

- for any $t \in(0,1 / 2)$ s.t. $\phi^{\prime}\left(\bar{D}^{-1}(t)\right) \geq 1$, we have $-\log t>\phi(0)$ and

$$
\bar{D}^{-1}(t) \leq \phi^{-1}(-\log t)
$$

If additionally $\phi$ satisfies $(B(\phi))$ and by letting $K=1+\frac{\phi^{\prime \prime}(1)}{\phi^{\prime}(1)^{2}}>0$, the following holds:

- for any $x>0$,

$$
\begin{aligned}
& \bar{D}(x) \geq \frac{d(x)}{\phi^{\prime}(x)}\left[1+\frac{\phi^{\prime \prime}(x)}{\phi^{\prime}(x)^{2}}\right]^{-1} \\
& \bar{D}(x) \geq \frac{d(x)}{\phi^{\prime}(x)} K^{-1} \quad \text { if } x \geq 1
\end{aligned}
$$

imsart-aos ver. 2012/08/16 file: AOS1042_supp.tex date: August 22, 2012 
- for any $t \in(0, \bar{D}(1))$ s.t. $\phi^{\prime}\left(\bar{D}^{-1}(t)\right) \geq 1$, we have $-\log t>\phi(0)$ and

$$
\bar{D}^{-1}(t) \geq \phi^{-1}\left(\phi(0) \vee\left\{-\log t-\log K-\log \circ \phi^{\prime} \circ \phi^{-1}(-\log t)\right\}\right) .
$$

Proof. First note that $\phi^{\prime}(x)>0$ in (S-44) because $\phi$ is increasing and convex. Next, (S-44) holds because $\phi^{\prime}$ is nondecreasing: $\bar{D}(x)=\int_{x}^{+\infty} e^{-\phi(u)} d u \leq$ $\left(\phi^{\prime}(x)\right)^{-1} \int_{x}^{+\infty} \phi^{\prime}(u) e^{-\phi(u)} d u=d(x) / \phi^{\prime}(x)$. Expression (S-45) follows from (S-44) applied with $x=\bar{D}^{-1}(t)$. To prove (S-46), write for any $x>0$,

$\frac{\phi^{\prime \prime}(x)}{\phi^{\prime}(x)^{2}} \bar{D}(x) \geq \int_{x}^{+\infty} \frac{\phi^{\prime \prime}(u)}{\phi^{\prime}(u)^{2}} e^{-\phi(u)} d u=\left[-\frac{e^{-\phi(u)}}{\phi^{\prime}(u)}\right]_{x}^{\infty}-\bar{D}(x)=\frac{d(x)}{\phi^{\prime}(x)}-\bar{D}(x)$,

by using an integration by parts. Expressions (S-46) and (S-47) follow. Finally, let us prove (S-48). From (S-47), we get $K t \phi^{\prime}\left(\bar{D}^{-1}(t)\right) \geq e^{-\phi\left(\bar{D}^{-1}(t)\right)}$ and thus $-\log (K t)-\log \circ \phi^{\prime}\left(\bar{D}^{-1}(t)\right) \leq \phi\left(\bar{D}^{-1}(t)\right)$. The result follows from $(\mathrm{S}-46)$.

\section{S-6. A sub-optimality result.}

Proposition S-6.1. Under Assumption $\left(\mathrm{A}\left(F_{m}, \tau_{m}\right)\right)$, let us choose $q_{m} \leq$ 1 (i.e., $\alpha_{m} \geq 1 / 2$ ) in the BFDR threshold $t_{m}^{\star}$. Then we have for any $m \geq 2$,

$$
R_{m}\left(t_{m}^{\star}\right) \geq R_{m}\left(t_{m}^{B}\right)\left(C_{m}\left(1 / q_{m}-1\right)+1\right) .
$$

In particular, under (BP) (and using $C_{-}$defined therein), if the sequence $\left(q_{m}\right)_{m}$ is such that $q_{m} \leq q_{+}<1$ (i.e., $\alpha_{m} \geq \alpha_{-}>1 / 2$ ) for all $m \geq 2$, we have for any $m \geq 2$,

$$
R_{m}\left(t_{m}^{\star}\right) / R_{m}\left(t_{m}^{B}\right) \geq C_{-}\left(1 / q_{+}-1\right)+1>1 .
$$

In particular, $t_{m}^{\star}$ is not asymptotically optimal.

Proof. First, since $F_{m}(t)=t \Psi_{m}(t)$

$$
\begin{aligned}
R_{m}\left(t_{m}^{B}\right) & =\pi_{0, m} t_{m}^{B}+\pi_{0, m} \tau_{m}^{-1}\left(1-t_{m}^{B} \Psi_{m}\left(t_{m}^{B}\right)\right) \\
& =\pi_{0, m} t_{m}^{B}\left(1-\tau_{m}^{-1} \Psi_{m}\left(t_{m}^{B}\right)\right)+\pi_{0, m} \tau_{m}^{-1} \\
& \leq \pi_{0, m} \tau_{m}^{-1},
\end{aligned}
$$

because $\Psi_{m}\left(t_{m}^{B}\right) \geq f_{m}\left(t_{m}^{B}\right)=\tau_{m}$ from the concavity of $F_{m}$.

Second, assuming $q_{m} \leq 1$, we have $\Psi_{m}\left(t_{m}^{B}\right) \geq \tau_{m} \geq q_{m} \tau_{m}=\Psi\left(t_{m}^{\star}\right)$. Hence $t_{m}^{B} \leq t_{m}^{\star}$ and $F_{m}\left(t_{m}^{\star}\right) \geq C$. By using (38), we get $R_{m}\left(t_{m}^{\star}\right) \geq \pi_{0, m} \tau_{m}^{-1}\left(C\left(1 / q_{m}-\right.\right.$ $1)+1)$, which, combined with (S-50), leads to (S-49). 
S-7. Additional numerical experiments. We provide the following additional experiments:

- Relative excess risks: we pictured the counterpart of Figure 4 in [2] in the Gaussian scale (Figure S-1), Laplace scale (Figure S-2) and Laplace location models (Figure S-3);

- Influence of $\left(\beta_{0}, C_{0}\right)$ on relative excess risk: we pictured the relative excess risk for FDR thresholding at level $\alpha_{m}^{o p t}\left(\beta_{0}, C_{0}\right)$ for three values of $\beta_{0}$ combined with three values of $C_{0}$, in the Gaussian location model (Figure S-4), in the Gaussian scale model (Figure S-5), Laplace scale model (Figure S-6) and Laplace location model (Figure S-7);

- Comparison between the performance of $\alpha_{m}^{o p t}\left(\beta_{0}, C_{0}\right)$ and $\alpha_{m}^{\infty}\left(\beta_{0}, C_{0}\right)$ : we pictured the case of a Gaussian location model (Figure S-8), Gaussian scale model (Figure S-9) and Laplace scale model (Figure S-10). We skipped the case of the Laplace location model because $\alpha_{m}^{o p t}\left(\beta_{0}, C_{0}\right)=$ $\alpha_{m}^{\infty}\left(\beta_{0}, C_{0}\right)=C_{0} /\left(1-C_{0}\right)$ in that case.

\section{References.}

[1] P. Neuvial. Intrinsic Bounds and False Discovery Rate Control in Multiple Testing Problems. hal-00460677 preprint.

[2] P. Neuvial and E. Roquain. On false discovery rate thresholding for classification under sparsity. Submitted, 2011.

Laboratoire Statistique et Génome UNIVERSité D'Évry VAL D'EsSONNE UMR CNRS 8071 - USC INRA

23 Boulevard de France, 91037 Évry, France

E-MAIL: pierre.neuvial@genopole.cnrs.fr
UPMC UNIV PARIS 6, LPMA, 4, Place Jussieu, 75252 Paris Cedex 05, France E-MAIL: etienne.roquain@upmc.fr 


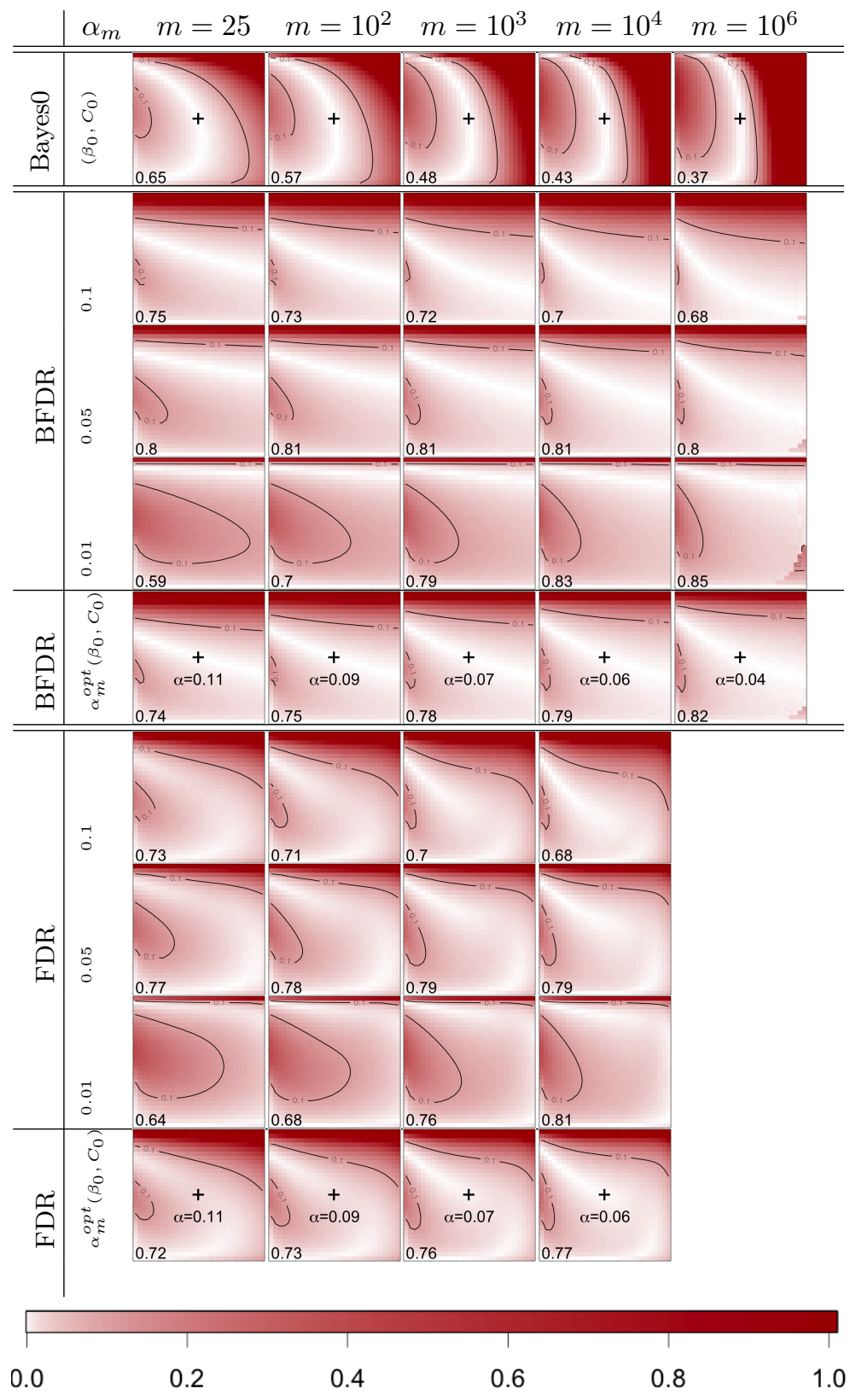

FIG S-1. Similar to Figure 4 for the Gaussian scale model. 


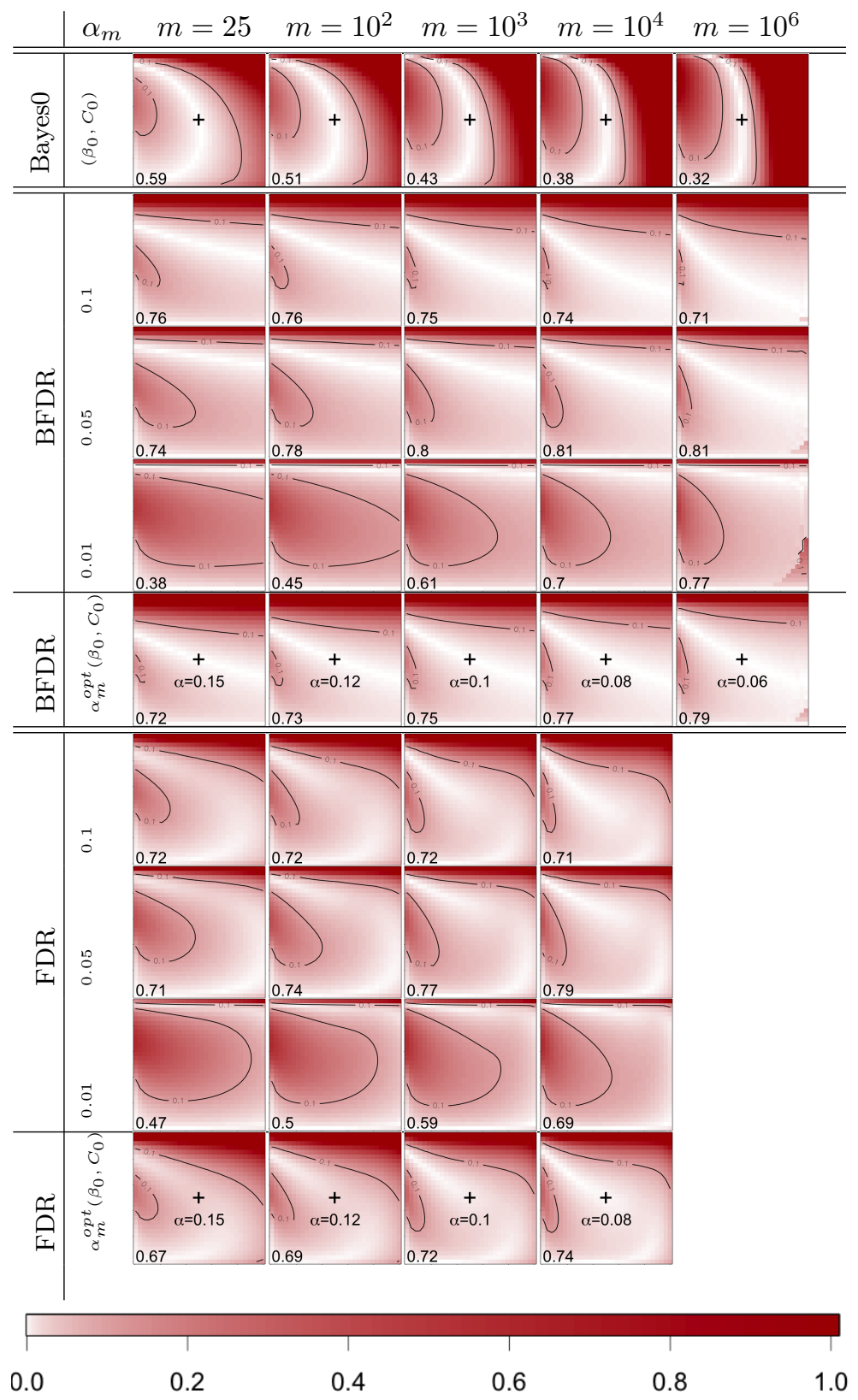

FIG S-2. Similar to Figure 4 for the Laplace scale model. 


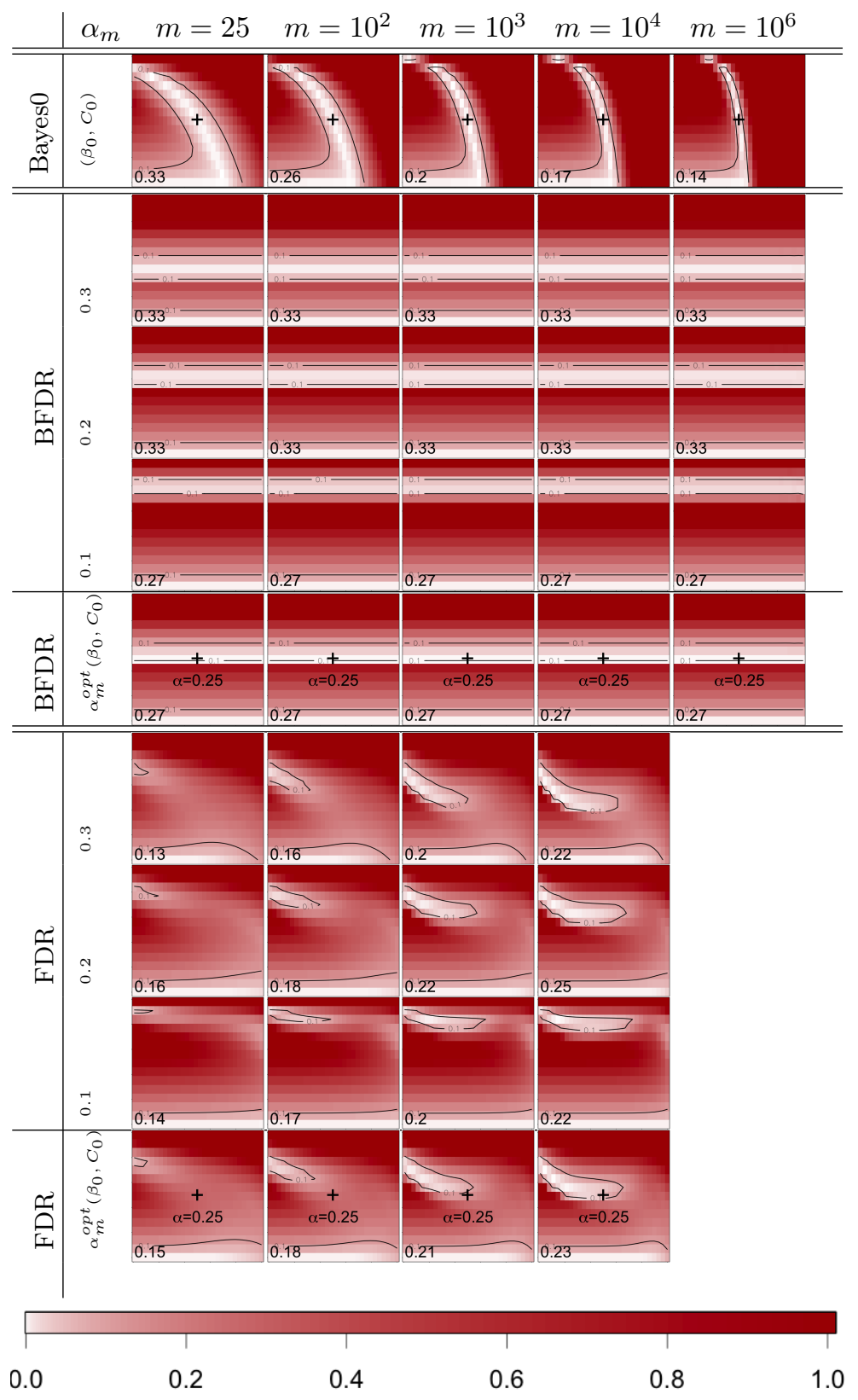

FIG S-3. Similar to Figure 4 for the Laplace location model. $C_{m}$ is taken larger than 0.5, see Section S-3.3. $\left(\beta_{0}, C_{0}\right)=(1 / 2,3 / 4)$. 


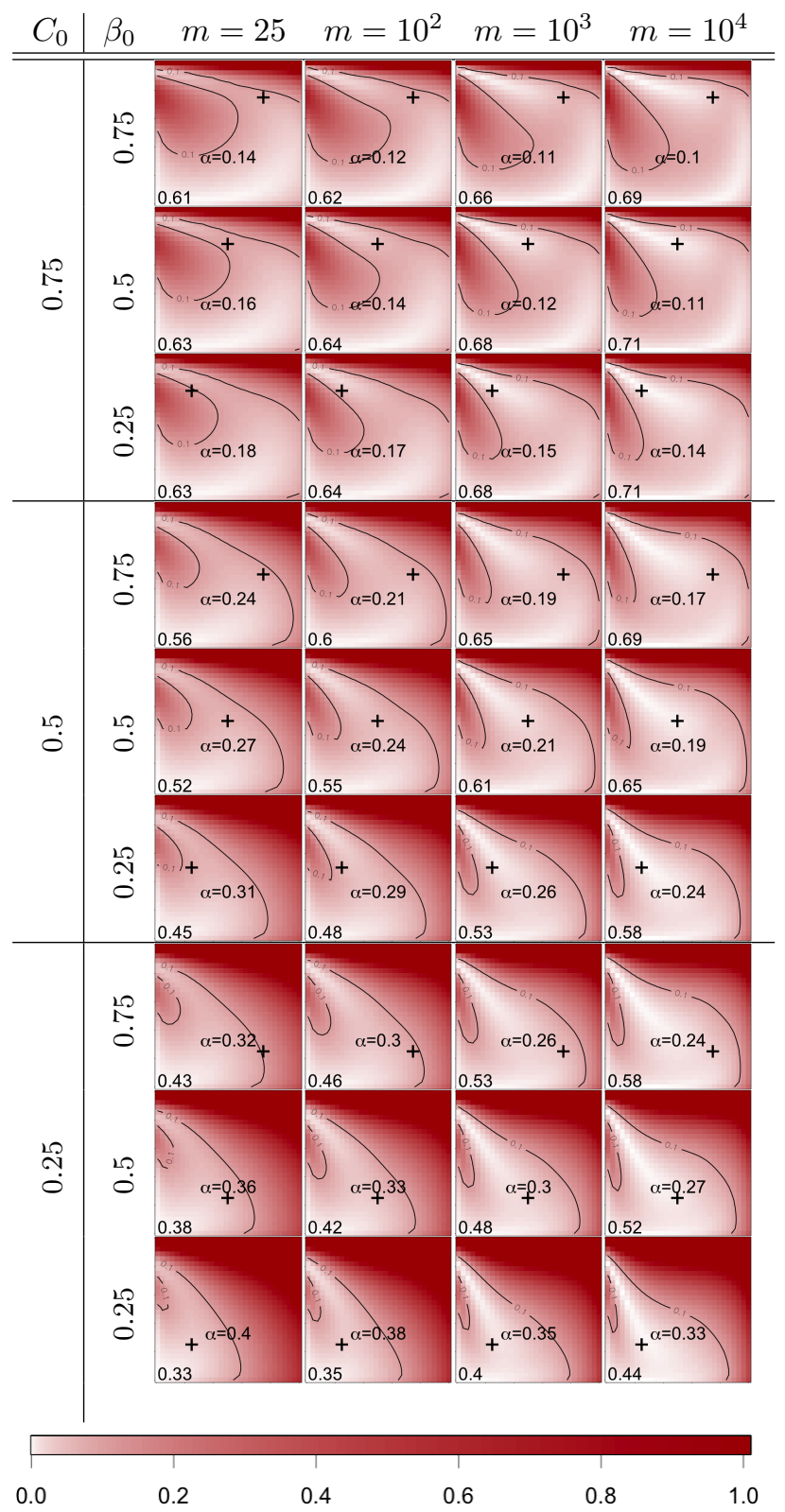

FIG S-4. Gaussian location model: relative excess risks $\left(\mathcal{E}_{m}\right)$ of FDR thresholding for $m \in\left\{25,100,10^{3}, 10^{4}\right\}, \beta_{0} \in\{0.25,0.5,0.75\}$ and $C_{0} \in\{0.25,0.5,0.75\}$. In each panel, the point $\left(\beta=\beta_{0}, C=C_{0}\right)$ is marked by " + ". 


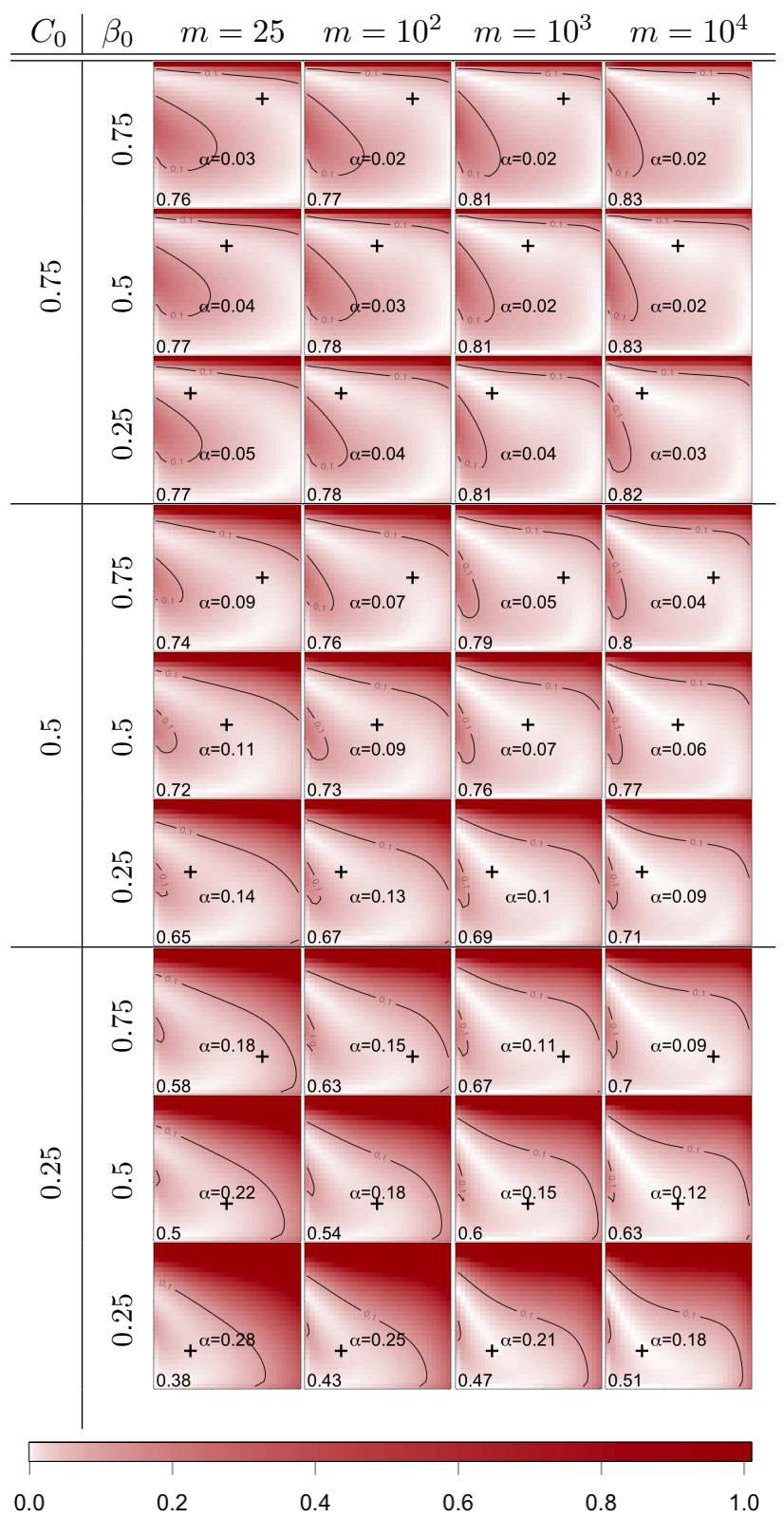

FIG S-5. Similar to Figure $S$-4 in the Gaussian scale model. 


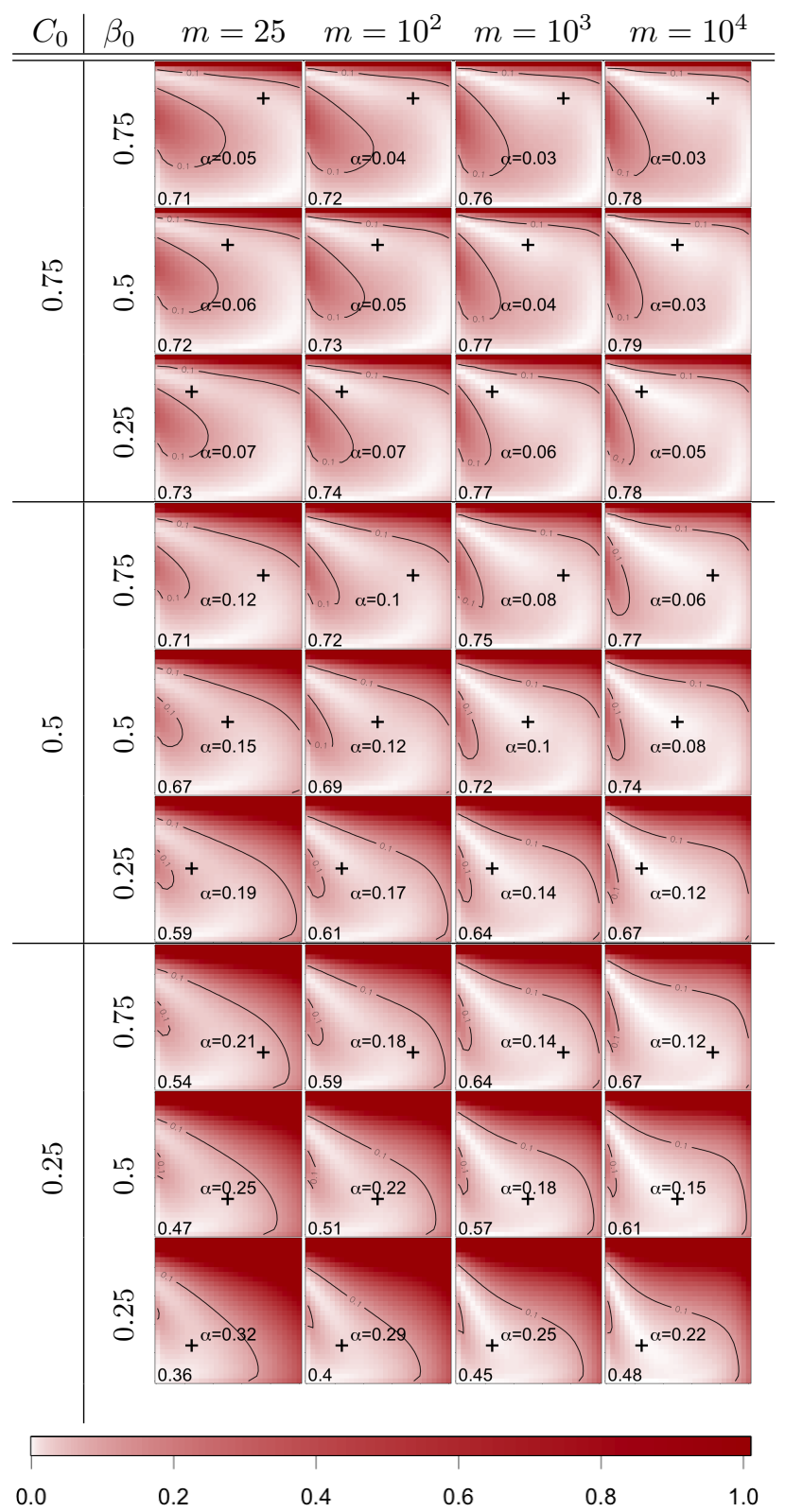

FIG S-6. Similar to Figure S-4 in the Laplace scale model. 


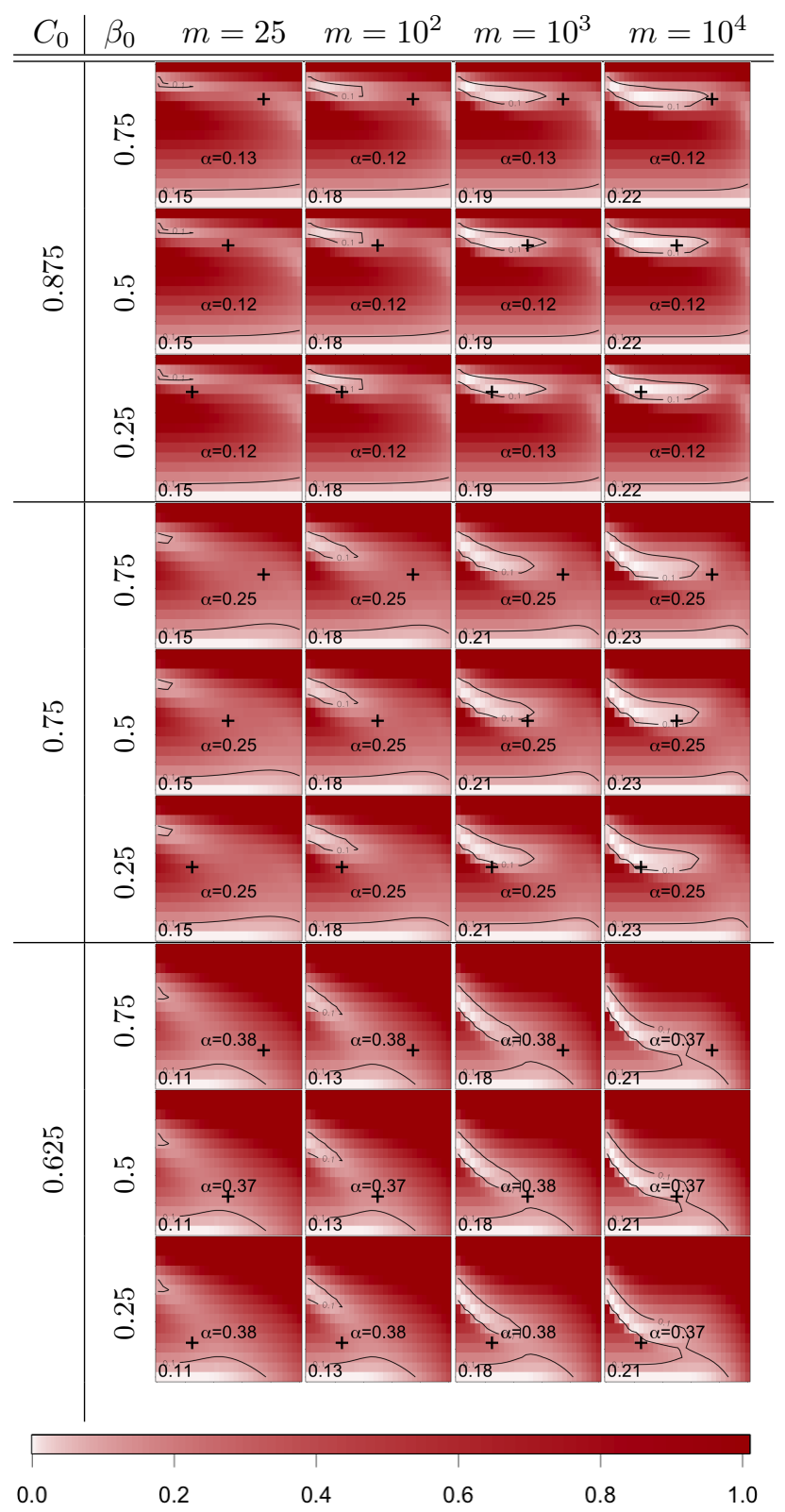

FIG S-7. Similar to Figure $S-4$ in the Laplace location model. $C_{m}$ is taken in the range $(1 / 2,1)$, see Section $S-3.3$, and $C_{0} \in\{0.625,0.75,0.875\}$. 


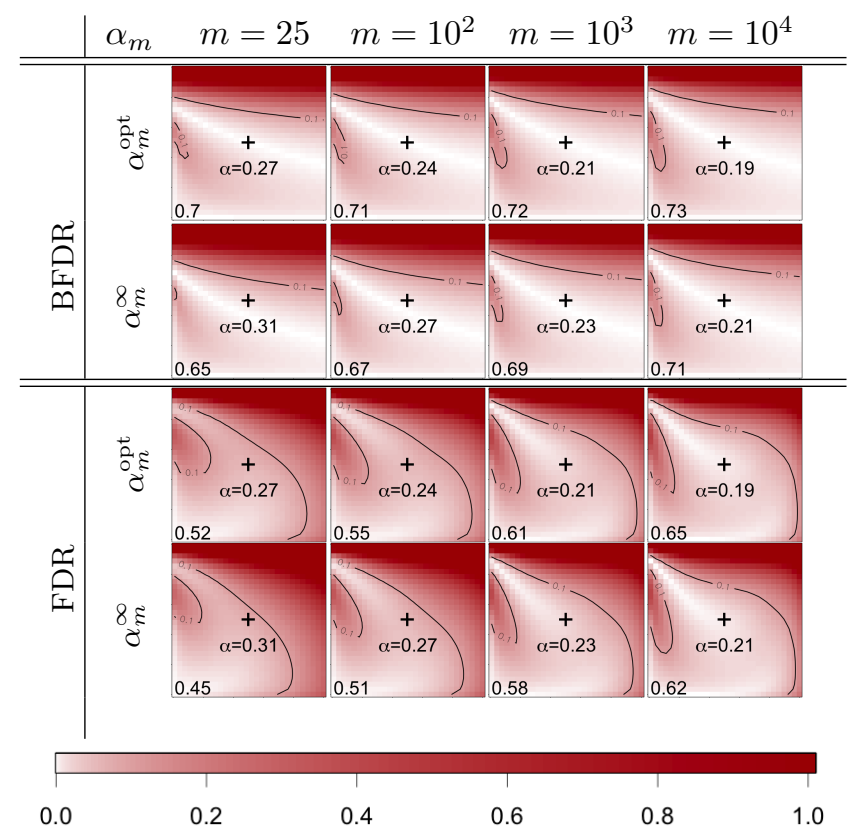

FIG S-8. Gaussian location model: excess risk ratios of BFDR and FDR thresholding for $m \in\{25,100,1000,10000\}$ for $\alpha_{m}^{o p t}\left(\beta_{0}=1 / 2, C_{0}=1 / 2\right)$ and $\alpha_{m}^{\infty}\left(\beta_{0}=1 / 2, C_{0}=1 / 2\right)$. 


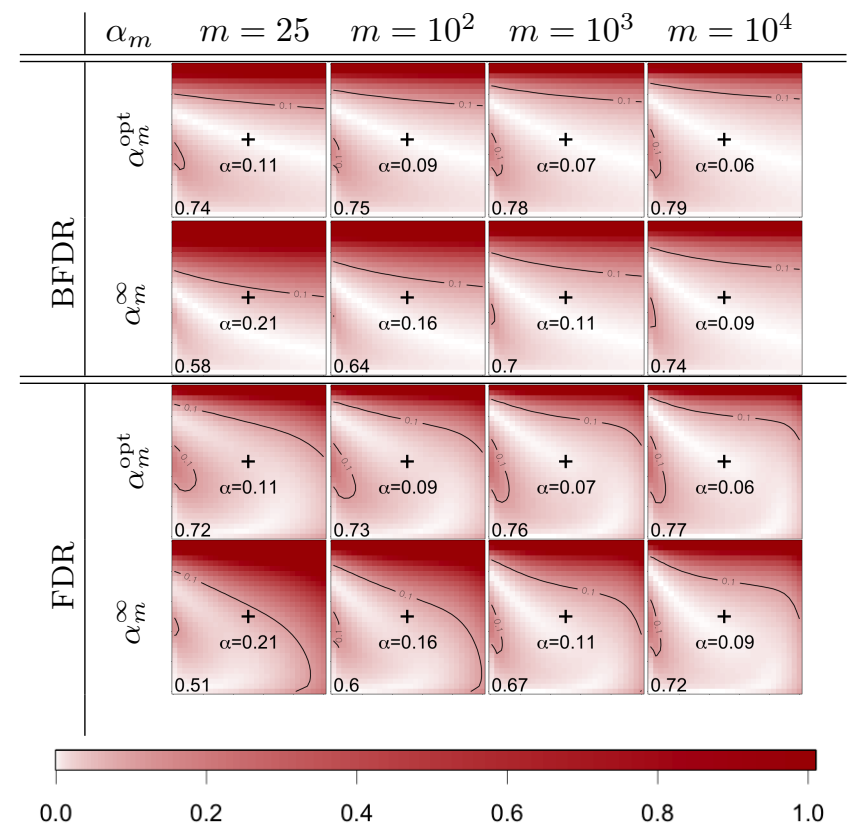

FIG S-9. Gaussian scale model: excess risk ratios of BFDR and FDR thresholding for $m \in\{25,100,1000,10000\}$ for $\alpha_{m}^{o p t}\left(\beta_{0}=1 / 2, C_{0}=1 / 2\right)$ and $\alpha_{m}^{\infty}\left(\beta_{0}=1 / 2, C_{0}=1 / 2\right)$. 


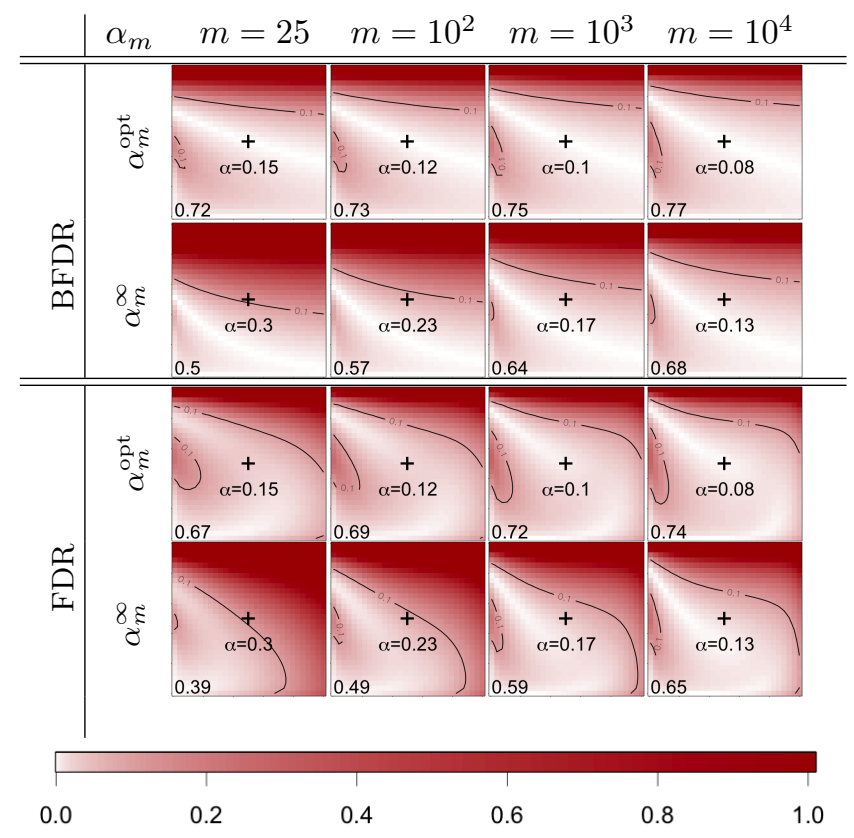

FIG S-10. Laplace scale model: excess risk ratios of BFDR and FDR thresholding for $m \in\{25,100,1000,10000\}$ for $\alpha_{m}^{o p t}\left(\beta_{0}=1 / 2, C_{0}=1 / 2\right)$ and $\alpha_{m}^{\infty}\left(\beta_{0}=1 / 2, C_{0}=1 / 2\right)$. 